\title{
Platinum-Catalyzed Intramolecular Alkylation of Indoles with Unactivated Olefins
}

Cong Liu, Xiaoqing Han, Xiang Wang, and Ross A. Widenhoefer*

P. M. Gross Chemical Laboratory

Duke University

Durham, NC 27708-0346

\section{Supporting Information}

Experimental procedures, analytical and spectroscopic data for indoles, copies of 2-D NMR spectra for anti-5- $d_{2}$, and X-ray crystal structure data for 5 (38 pages). 


\section{Experimental}

General Methods. Catalytic reactions were performed in a thick-walled pressure tube (Ace Glass) under a nitrogen atmosphere. NMR spectra were obtained on a Varian spectrometer operating at $400 \mathrm{MHz}$ for ${ }^{1} \mathrm{H} \mathrm{NMR}$ and $100 \mathrm{MHz}$ for ${ }^{13} \mathrm{C} \mathrm{NMR}$ in $\mathrm{CDCl}_{3}$ unless otherwise noted. IR spectra were obtained on a Bomen MB-100 FT IR spectrometer. Gas chromatography was performed on a Hewlett-Parkard 5890 gas chromatography equipped with a $25 \mathrm{~m}$ polydimethylsiloxane capillary column. Flash column chromatography was performed employing 200-400 mesh silica gel (EM). Thin layer chromatography (TLC) was performed on silica gel $60 \mathrm{~F}_{254}$. Elemental analyses were performed by Complete Analysis Laboratories (Parsippany, NJ). 1,4-Dioxane (Acros, anhydrous) and $\mathrm{HCl}$ solution (4.0 M, 1,4-dioxane, Aldrich) were stored in an inert atmosphere glovebox. $\left[\mathrm{PtCl}_{2}\left(\mathrm{H}_{2} \mathrm{C}=\mathrm{CH}_{2}\right)\right]_{2}$, $\mathrm{PtCl}_{2}(\mathrm{MeCN})_{2}$ and $\mathrm{PtCl}_{2}$ (Strem), methyl 4-pentenoate, ethyl 5-methyl-5-hexenoate, cis-2penten-1-ol, trans-2-penten-1-ol, 1-methyl-1H-indole-2-carboxylic acid (Aldrich), and 3chloro-1-cyclohexene (TCI) were used as received. . N-Trimethylsilyl-o-toluidine (S1), ${ }^{1}$ 2-(3butenyl)-1H-indole, ${ }^{2}$ methyl 2,2-dimethyl-5-hexenoate, ${ }^{3}$ methyl 2,2-dimethyl-4-pentenoate, ${ }^{4}$ 2-hydroxymethyl-1-methyl-1H-indole $(\mathbf{S 2}){ }^{5} \quad \mathrm{~N}$-allyl- $N$-methyl $1 H$-indole-2-carboxamide, ${ }^{6}$ and 1,3-dideuterio-2-cyclohexen-1-ol ${ }^{7}$ were synthesized employing published procedures. (R)-6,2'-bis-[bis-(3,5-di-tert-butyl-4-methoxyphenyl)-phosphino]-2,6'-dimethoxybiphenyl [(R)-S3] and (R)-3,5-i-Pr-MeOBIPHEP were provided by Dr. Rudolph Schmid (HoffmannLaRoche).

\section{2-Alkenyl Indoles}

1-Methyl-2-(4-pentenyl)-1H-indole (1). $n$-BuLi (2.5 M solution in hexanes, $26.0 \mathrm{~mL}$, $65.0 \mathrm{mmol}$ ) was added dropwise to a solution of $\mathbf{S 1}(4.70 \mathrm{~g}, 26.0 \mathrm{mmol})$ in hexanes (150 
$\mathrm{mL}$ ) at $0{ }^{\circ} \mathrm{C}$. The resulting orange solution was refluxed for $6 \mathrm{~h}$, cooled to $-78{ }^{\circ} \mathrm{C}$, and treated with a solution of methyl 5-hexenoate $(6.25 \mathrm{~g}, 44.0 \mathrm{mmol})$ in THF $(25 \mathrm{~mL})$. The resulting mixture was warmed to room temperature, stirred for $1 \mathrm{~h}$, and quenched with brine. The aqueous layer was extracted with ether $(2 \times 100 \mathrm{~mL})$ and the layers were separated. The combined organic extracts were dried $\left(\mathrm{MgSO}_{4}\right)$ and concentrated under vacuum. Column chromatography of the residue $\left(\mathrm{SiO}_{2}\right.$; hexanes- $\left.\mathrm{CH}_{2} \mathrm{Cl}_{2}=5: 1\right)$ gave 2-(4-pentenyl)-1 $\mathrm{H}$-indole (S4) (2.99 g, 62\%) as a yellow oil. Compound S4 (196 mg, $1.1 \mathrm{mmol})$ and MeI (137 $\mu \mathrm{L}, 2.2$ mmol) were added sequentially to a suspension of $\mathrm{KOH}(560 \mathrm{mg}, 10.0 \mathrm{mmol})$ in acetone (5 $\mathrm{mL}$ ). The resulting mixture was stirred at room temperature for $3 \mathrm{~h}$ and quenched with water. The layers were separated and the aqueous layer was extracted with ether $(3 \times 10 \mathrm{~mL})$. The combined organic extracts were washed (brine), dried $\left(\mathrm{MgSO}_{4}\right)$, and concentrated under vacuum. Column chromatography of the residue $\left(\mathrm{SiO}_{2}\right.$; hexanes- $\left.\mathrm{CH}_{2} \mathrm{Cl}_{2}=30: 1\right)$ gave $\mathbf{1}(162$ $\mathrm{mg}, 77 \%$ ) as a pale yellow oil.

For S4: $\mathrm{TLC}$ (hexanes- $\left.\mathrm{CH}_{2} \mathrm{Cl}_{2}=1: 1\right): R_{f}=0.59 .{ }^{1} \mathrm{H} \mathrm{NMR}: \delta \quad 7.80(\mathrm{~s}, 1 \mathrm{H}), 7.59-7.57$ $(\mathrm{m}, 1 \mathrm{H}), 7.32-7.29(\mathrm{~m}, 1 \mathrm{H}), 7.16(\mathrm{dt}, J=1.6,7.6 \mathrm{~Hz}, 1 \mathrm{H}), 7.12(\mathrm{dt}, J=1.2,7.4 \mathrm{~Hz}, 1 \mathrm{H})$, 6.29-6.28 (m, $1 \mathrm{H}), 5.88(\mathrm{tdd}, J=6.8,10.4,17.2 \mathrm{~Hz}, 1 \mathrm{H}), 5.13-5.04(\mathrm{~m}, 2 \mathrm{H}), 2.78(\mathrm{t}, J=7.6$ $\mathrm{Hz}, 2 \mathrm{H}), 2.19(\mathrm{q}, J=7.2 \mathrm{~Hz}, 2 \mathrm{H}), 1.85$ (quint, $J=7.2 \mathrm{~Hz}, 2 \mathrm{H}) .{ }^{13} \mathrm{C}\left\{{ }^{1} \mathrm{H}\right\} \mathrm{NMR}: \delta$ 139.8, $138.5,136.1,129.1,121.3,120.1,119.9,115.5,110.6,99.9,33.5,28.6,27.9$. IR (neat, $\left.\mathrm{cm}^{-1}\right)$ : 3404, 2931, 1456, 1415, 1287, 911, 781, 749. Anal. calcd (found) for $\mathrm{C}_{13} \mathrm{H}_{15} \mathrm{~N}$ : C, 84.28 (84.21); H, $8.16(8.11)$.

For 1: $\mathrm{TLC}$ (hexanes- $\mathrm{CH}_{2} \mathrm{Cl}_{2}=5: 1$ ): $R_{f}=0.68 .{ }^{1} \mathrm{H} \mathrm{NMR}: \delta 7.58(\mathrm{br} \mathrm{d}, J=7.6 \mathrm{~Hz}, 1$ H), $7.30(\mathrm{dd}, J=0.8,8.0 \mathrm{~Hz}, 1 \mathrm{H}), 7.20(\mathrm{ddd}, J=1.2,7.6,8.0 \mathrm{~Hz}, 1 \mathrm{H}), 7.11(\mathrm{dt}, J=1.2,7.8$ $\mathrm{Hz}, 1 \mathrm{H}), 6.30(\mathrm{~d}, J=0.8 \mathrm{~Hz}, 1 \mathrm{H}), 5.91(\mathrm{tdd}, J=6.8,10.4,17.2 \mathrm{~Hz}, 1 \mathrm{H}), 5.15-5.05$ (m, 2 H), $3.69(\mathrm{~s}, 3 \mathrm{H}), 2.79(\mathrm{t}, J=7.6 \mathrm{~Hz}, 2 \mathrm{H}), 2.25$ (tq, $J=1.2,6.8 \mathrm{~Hz}, 2 \mathrm{H}), 1.87$ (quint, $J$ 
$=7.6 \mathrm{~Hz}, 2 \mathrm{H}) .{ }^{13} \mathrm{C}\left\{{ }^{1} \mathrm{H}\right\}$ NMR: $\delta 141.3,138.5,137.7,128.2,120.8,120.1,119.5,115.5$, 109.0, 99.1, 33.7, 29.7, 28.1, 26.5. IR (neat, $\left.\mathrm{cm}^{-1}\right)$ : 3060, 2933, 1456, 1312, 911, 772, 747. Anal. calcd (found) for $\mathrm{C}_{14} \mathrm{H}_{17} \mathrm{~N}: \mathrm{C}, 84.37$ (84.14); H, 8.60 (8.76).

1-Benzyl-2-(4-pentenyl)-1 $\mathbf{H}$-indole (Table 1, entry 2). A solution of S4 (0.66 g, 3.5 $\mathrm{mmol})$ in DMF (5 mL) was added to a suspension of $\mathrm{NaH}(85 \mathrm{mg}, 3.5 \mathrm{mmol})$ in DMF (5 mL) at $0{ }^{\circ} \mathrm{C}$. The resulting mixture was stirred for $10 \mathrm{~min}$ and treated with a solution of benzylchloride $(0.45 \mathrm{~g}, 3.5 \mathrm{mmol})$ in DMF ( $3 \mathrm{~mL})$. The resulting suspension was warmed to room temperature, stirred for $4 \mathrm{~h}$, and quenched with aqueous $1 \mathrm{~N} \mathrm{HCl}$. The layers were separated and the aqueous layer was extracted with ether $(3 \times 10 \mathrm{~mL})$. The combined organic extracts were washed (brine), dried $\left(\mathrm{MgSO}_{4}\right)$, and concentrated under vacuum. Column chromatography of the residue $\left(\mathrm{SiO}_{2}\right.$; hexanes-ether $=25: 1)$ gave 1-benzyl-2-(4-pentenyl)-1 $H$-indole $(0.89 \mathrm{~g}, 73 \%)$ as a yellow oil. TLC (hexanes-EtOAc $=5: 1): \quad R_{f}=0.72 .{ }^{1} \mathrm{H}$ NMR: $\delta 7.66-7.63(\mathrm{~m}, 1 \mathrm{H}), 7.32-7.20(\mathrm{~m}, 5 \mathrm{H})$, 7.17-7.12 (m, 2 H), 7.00-6.98 (m, 2 H), 6.41 (d, $J=0.8 \mathrm{~Hz}, 1 \mathrm{H}), 5.84$ (tdd, $J=6.8,10.4,17.2$ $\mathrm{Hz}, 1 \mathrm{H}), 5.34$ (s, $2 \mathrm{H}), 5.09-5.00$ (m, $2 \mathrm{H}), 2.73$ (t, $J=7.6 \mathrm{~Hz}, 2 \mathrm{H}), 2.19$ (tq, $J=1.2,6.8 \mathrm{~Hz}, 2$ H), 1.84 (quint, $J=7.6 \mathrm{~Hz}, 2 \mathrm{H}$ ). ${ }^{13} \mathrm{C}\left\{{ }^{1} \mathrm{H}\right\}$ NMR: $\delta 141.2,138.3,137.4,128.9,128.5,128.3$, 127.4, 126.1, 121.0, 120.0, 119.7, 115.3, 109.5, 99.7, 46.6, 33.5, 27.7, 26.2. IR (neat, $\mathrm{cm}^{-1}$ ): $3050,3027,2926,2860,1494,1465,1452,1314,735,696$. HRMS calcd (found) for $\mathrm{C}_{20} \mathrm{H}_{21} \mathrm{~N}$ $\left(\mathrm{M}^{+}\right): 275.1674$ (275.1684).

5-Methoxy-1-methyl-2-(4-pentenyl)-1H-indole (Table 1 , entry 3). 5-Methoxy-2-(4pentenyl)-1 $H$-indole (S5) was isolated in 30\% yield as a yellow oil from reaction of $\mathbf{S 1}$ and methyl 5-hexenoate employing a procedure similar to that used to synthesize $\mathbf{S 4}$. 5-Methoxy-1methyl-2-(4-pentenyl)-1 $\mathrm{H}$-indole was isolated in 53\% yield as a pale yellow oil from $\mathbf{S 5}$ and methyl iodide employing a procedure similar to that used to synthesize $\mathbf{1}$.

For S5: $\quad$ TLC (hexanes- $\left.\mathrm{CH}_{2} \mathrm{Cl}_{2}=1: 2\right): \quad R_{f}=0.44 .{ }^{1} \mathrm{H}$ NMR: $\delta 7.79(\mathrm{~s}, 1 \mathrm{H}), 7.18(\mathrm{dd}, J$ 
$=0.8,8.8 \mathrm{~Hz}, 1 \mathrm{H}), 7.04(\mathrm{~d}, J=2.4 \mathrm{~Hz}, 1 \mathrm{H}), 6.80(\mathrm{ddd}, J=0.8,2.8,8.8 \mathrm{~Hz}, 1 \mathrm{H}), 6.20-6.19(\mathrm{~m}$, $1 \mathrm{H}), 5.85$ (tdd, $J=6.8,10.4,17.2 \mathrm{~Hz}, \mathrm{H}), 5.10-5.00(\mathrm{~m}, 2 \mathrm{H}), 3.86(\mathrm{~s}, 3 \mathrm{H}), 2.74(\mathrm{t}, J=7.6 \mathrm{~Hz}, 2$ H), 2.16 (q, $J=7.2 \mathrm{~Hz}, 2 \mathrm{H}$ ), 1.82 (quint, $J=7.2 \mathrm{~Hz}, 2 \mathrm{H}$ ). ${ }^{13} \mathrm{C}\left\{{ }^{1} \mathrm{H}\right\}$ NMR: $\delta$ 154.4, 140.7, $138.5,131.3,129.6,115.4,111.3,111.1,102.3,99.8,56.2,33.5,28.6,27.9$. IR (neat, $\mathrm{cm}^{-1}$ ): $3405,2936,1456,1588,1485,1453,1168,1032,913$. HRMS calcd (found) for $\mathrm{C}_{14} \mathrm{H}_{17} \mathrm{O}_{\mathrm{N}} \mathrm{O}$ $\left(\mathrm{M}^{+}\right): 215.1310(215.1310)$.

For 5-Methoxy-1-methyl-2-(4-pentenyl)-1H-indole: TLC (hexanes- $\left.\mathrm{CH}_{2} \mathrm{Cl}_{2}=1: 2\right): R_{f}=$ 0.59. ' ${ }^{1} \mathrm{H}$ NMR: $\delta 7.18(\mathrm{br} \mathrm{d}, J=8.8 \mathrm{~Hz}, 1 \mathrm{H}), 7.06(\mathrm{br} \mathrm{d}, J=2.4 \mathrm{~Hz}, 1 \mathrm{H}), 6.84(\mathrm{dd}, J=2.4,8.8$ Hz, $1 \mathrm{H}), 6.22$ (s, $1 \mathrm{H}), 5.89$ (tdd, $J=6.4,10.4,17.2 \mathrm{~Hz}, 1 \mathrm{H}), 5.14-5.04$ (m, $2 \mathrm{H}), 3.87$ (s, $3 \mathrm{H}$ ), 3.65 (s, $3 \mathrm{H}$ ), 2.75 (t, $J=7.8 \mathrm{~Hz}, 2 \mathrm{H}), 2.23$ (q, $J=7.2 \mathrm{~Hz}, 2 \mathrm{H}$ ), 1.85 (quint, $J=7.6 \mathrm{~Hz}, 2 \mathrm{H}$ ). ${ }^{13} \mathrm{C}\left\{{ }^{1} \mathrm{H}\right\}$ NMR: $\delta 154.3,141.9,138.5,133.0,128.5,115.5,110.7,109.7,102.3,98.8,56.3,33.6$, 29.8, 28.1, 26.5. IR (neat, $\mathrm{cm}^{-1}$ ): 2936, 1620, 1486, 1403, 1214, 1147, 1033, 911. Anal. calcd (found) for $\mathrm{C}_{15} \mathrm{H}_{19} \mathrm{NO}: \mathrm{C}, 78.56$ (78.74); $\mathrm{H}, 8.35$ (8.37).

cis-2-(2,2-Dicarbomethoxy-4-heptenyl)-1-methyl-1H-indole (Table 1, entry 4). $n$-BuLi (2.5 M solution in hexanes, $10.5 \mathrm{~mL}, 26.3 \mathrm{mmol})$ was added to a solution of $\mathbf{S 2}(4.03 \mathrm{~g}, 25.0$ $\mathrm{mmol})$ in $\mathrm{THF}(50 \mathrm{~mL})$ at $-78^{\circ} \mathrm{C}$. After $2 \mathrm{~min}, \mathrm{MsCl}(3.11 \mathrm{~g}, 27.5 \mathrm{mmol})$ and $\mathrm{LiBr}(6.53 \mathrm{~g}, 75.0$ mmol) were added sequentially. The resulting mixture was warmed at $0{ }^{\circ} \mathrm{C}$, stirred for $30 \mathrm{~min}$, and transferred via cannula to a solution of sodium dimethyl malonate in THF [generated from dimethyl malonate $(6.60 \mathrm{~g}, 50.0 \mathrm{mmol})$ and $\mathrm{NaH}(1.20 \mathrm{~g}, 50.0 \mathrm{mmol})$ in THF $(100 \mathrm{~mL})]$. The resulting mixture was stirred at room temperature for $2 \mathrm{~h}$ and quenched with water. The layers were separated and the aqueous layer was extracted with ether $(3 \times 150 \mathrm{~mL})$. The combined organic extracts were washed (brine), dried $\left(\mathrm{MgSO}_{4}\right)$ and concentrated under vacuum. Column chromatography of the residue $\left(\mathrm{SiO}_{2}\right.$; hexanes-EtOAc = 5:1) gave dimethyl 2-(1-methyl-1Hindol-2-ylmethyl)malonate (S6) $(2.54 \mathrm{~g}, 37 \%)$ as a pale yellow solid. $n$-BuLi (2.5 M solution 
in hexanes, $5.1 \mathrm{~mL}, 12.6 \mathrm{mmol})$ was added to a solution of $c i s-2$-penten-1-ol (1.03 g, $12.0 \mathrm{mmol})$ in THF $(10 \mathrm{~mL})$ at $-78^{\circ} \mathrm{C}$. After stirring for $2 \mathrm{~min}, \mathrm{MsCl}(1.49 \mathrm{~g}, 13.2 \mathrm{mmol})$ and $\mathrm{LiBr}(3.13 \mathrm{~g}$, $36.0 \mathrm{mmol}$ ) were added sequentially. The resulting mixture was warmed to $0{ }^{\circ} \mathrm{C}$, stirred for 30 min, and transferred via cannula to a solution of sodium dimethyl 2-(1-methyl-1H-indol-2ylmethyl)malonate [prepared from S6 (1.10 g, $4.0 \mathrm{mmol})$ and $\mathrm{NaH}(97 \mathrm{mg}, 4.05 \mathrm{mmol})$ in THF $(10 \mathrm{~mL})]$. The resulting mixture was stirred at room temperature for $3 \mathrm{~h}$ and quenched with water. The layers were separated and the aqueous layer was extracted with ether $(3 \times 30 \mathrm{~mL})$. The combined organic extracts were washed (brine), dried $\left(\mathrm{MgSO}_{4}\right)$, and concentrated under vacuum. Column chromatography of the residue $\left(\mathrm{SiO}_{2}\right.$; hexanes-EtOAc $\left.=5: 1\right)$ gave cis-2-(2,2dicarbomethoxy-4-heptenyl)-1-methyl-1H-indole $(0.75 \mathrm{~g}, 55 \%)$ as a viscous yellow oil.

For S6: $\operatorname{mp} 87-89{ }^{\circ} \mathrm{C} . \quad$ TLC (hexanes-EtOAc $=2: 1$ ): $R_{f}=0.45 .{ }^{1} \mathrm{H}$ NMR: $\delta 7.53(\mathrm{br} \mathrm{d}, J$ $=8.0 \mathrm{~Hz}, 1 \mathrm{H}), 7.28(\mathrm{br} \mathrm{d}, J=8.4,1 \mathrm{H}), 7.18(\mathrm{br} \mathrm{t}, J=7.2 \mathrm{~Hz}, 1 \mathrm{H}), 7.10-7.06(\mathrm{~m}, 1 \mathrm{H}), 6.27(\mathrm{~s}$, $1 \mathrm{H}), 3.88(\mathrm{t}, J=7.6 \mathrm{~Hz}, 1 \mathrm{H}), 3.76(\mathrm{~s}, 6 \mathrm{H}), 3.71(\mathrm{~s}, 3 \mathrm{H}), 3.41(\mathrm{~d}, J=7.6 \mathrm{~Hz}, 2 \mathrm{H}) .{ }^{13} \mathrm{C}\left\{{ }^{1} \mathrm{H}\right\}$ NMR: $\delta 169.3,137.8,136.9,128.0,121.5,120.4,119.8,109.3,99.9,53.2,51.4,29.8,26.3$. IR (neat, $\mathrm{cm}^{-1}$ ): 2949, 1757, 1732, 1649, 1434, 749. Anal. calcd (found) for $\mathrm{C}_{15} \mathrm{H}_{17} \mathrm{NO}_{4}$ : C, 65.44 (65.22); H, 6.22 (6.17).

For cis-2-(2,2-Dicarbomethoxy-4-heptenyl)-1-methyl-1H-indole: TLC (hexanes-EtOAc = 2:1): $R_{f}=0.64 .{ }^{1} \mathrm{H}$ NMR: $\delta 7.53($ br d $, J=7.6 \mathrm{~Hz}, 1 \mathrm{H}), 7.27($ br d, $J=8.0 \mathrm{~Hz}, 1 \mathrm{H}), 7.18(\mathrm{dt}, J$ = 1.2, 7.6 Hz, $1 \mathrm{H}), 7.10-7.06(\mathrm{~m}, 1 \mathrm{H}), 6.26(\mathrm{~s}, 1 \mathrm{H}), 5.60-5.53(\mathrm{~m}, 1 \mathrm{H}), 5.31-5.23(\mathrm{~m}, 1 \mathrm{H})$, $3.74(\mathrm{~s}, 6 \mathrm{H}), 3.67(\mathrm{~s}, 3 \mathrm{H}), 3.45(\mathrm{~s}, 2 \mathrm{H}), 2.82(\mathrm{dd}, J=1.0,7.4 \mathrm{~Hz}, 2 \mathrm{H}), 1.94$ (d quint, $J=1.2$, $7.4 \mathrm{~Hz}, 2 \mathrm{H}), 0.89(\mathrm{t}, J=7.6 \mathrm{~Hz}, 3 \mathrm{H}) .{ }^{13} \mathrm{C}\left\{{ }^{1} \mathrm{H}\right\} \mathrm{NMR}: \delta \quad 171.7,137.5,136.5,135.1,128.1$, $122.1,121.4,120.4,119.8,109.3,101.2,58.0,53.0,30.3,30.0,29.3,20.9,14.3$. IR (neat, $\mathrm{cm}^{-1}$ ): 2954, 1737, 1468, 1434, 1318, 1289, 1214, 750. Anal. calcd (found) for $\mathrm{C}_{20} \mathrm{H}_{25} \mathrm{NO}_{4}$ : C, 69.95 (69.94); H, 8.16 (8.29). 
trans-2-(2,2-Dicarbomethoxy-4-heptenyl)-1-methyl-1H-indole (Table 1, entry 5). trans2-(2,2-Dicarbomethoxy-4-heptenyl)-1-methyl-1H-indole was isolated in $81 \%$ yield as a viscous yellow oil from reaction of trans-2-penten-1-ol and S6 employing a procedure similar to that used to synthesize cis-2-(2,2-dicarbomethoxy-4-heptenyl)-1-methyl-1H-indole. TLC (hexanes-EtOAc $=2: 1): R_{f}=0.68 .{ }^{1} \mathrm{H}$ NMR: $\delta 7.55-7.53(\mathrm{~m}, 1 \mathrm{H}), 7.28(\mathrm{dd}, J=0.8,8.0 \mathrm{~Hz}, 1$ H), $7.19(\mathrm{dt}, J=1.2,7.6 \mathrm{~Hz}, 1 \mathrm{H}), 7.09$ (dt, $J=1.2,8.0 \mathrm{~Hz}, 1 \mathrm{H}), 6.27$ (d, $J=0.8 \mathrm{~Hz}, 1 \mathrm{H}), 5.58$ $5.51(\mathrm{~m}, 1 \mathrm{H}), 5.37-5.29(\mathrm{~m}, 1 \mathrm{H}), 3.74(\mathrm{~s}, 6 \mathrm{H}), 3.68(\mathrm{~s}, 3 \mathrm{H}), 3.43(\mathrm{~s}, 2 \mathrm{H}), 2.76(\mathrm{dd}, J=1.0,7.4$ $\mathrm{Hz}, 2 \mathrm{H}), 2.06-1.99$ (m, $2 \mathrm{H}), 0.97$ (t, $J=7.4 \mathrm{~Hz}, 3 \mathrm{H}) .{ }^{13} \mathrm{C}\left\{{ }^{1} \mathrm{H}\right\} \mathrm{NMR}: \delta \quad 171.6,137.8,137.5$, $135.2,128.1,122.7,121.4,120.4,119.8,109.4,101.2,58.5,52.9,36.0,30.0,29.3,26.0,14.1$. IR (neat, $\mathrm{cm}^{-1}$ ): 2955, 1737, 1468, 1434, 1210, 971, 749. Anal. calcd (found) for $\mathrm{C}_{20} \mathrm{H}_{25} \mathrm{NO}_{4}$ : C, 69.95 (69.80); H, 7.34 (7.28).

1-Methyl-2-(4-methyl-4-pentenyl)-1H-indole (Table 1, entry 6). 2-(4-Methyl-4pentenyl)-1 $H$-indole (S7) was isolated in $45 \%$ yield as a yellow oil from reaction of $\mathbf{S 1}$ and ethyl 5-methyl-5-hexenoate employing a procedure similar to that used to synthesize S4. 1-Methyl-2(4-methyl-4-pentenyl)- $1 H$-indole was isolated in $80 \%$ yield as a yellow oil from reaction of $\mathbf{S 7}$ and methyl iodide employing a procedure similar to that used to synthesize $\mathbf{1}$.

For S7: $\mathrm{TLC}$ (hexanes- $\left.\mathrm{CH}_{2} \mathrm{Cl}_{2}=1: 1\right): R_{f}=0.57 .{ }^{1} \mathrm{H}$ NMR: $\delta 7.82(\mathrm{~s}, 1 \mathrm{H}), 7.58-7.56(\mathrm{~m}$, $1 \mathrm{H}), 7.32-7.30(\mathrm{~m}, 1 \mathrm{H}), 7.18-7.09$ (m, $2 \mathrm{H}), 6.28$ (dd, $J=0.8,2.0 \mathrm{~Hz}, 1 \mathrm{H}), 4.81-4.80(\mathrm{~m}, 1 \mathrm{H})$, 4.76-4.75 (m, $1 \mathrm{H}), 2.76(\mathrm{t}, J=7.4 \mathrm{~Hz}, 2 \mathrm{H}), 2.15(\mathrm{t}, J=7.4 \mathrm{~Hz}, 2 \mathrm{H}), 1.89$ (quint, $J=7.4 \mathrm{~Hz}, 2$ $\mathrm{H}), 1.78(\mathrm{~s}, 6 \mathrm{H}) .{ }^{13} \mathrm{C}\left\{{ }^{1} \mathrm{H}\right\} \mathrm{NMR}: \delta 145.6,139.9,136.1,129.1,121.3,120.1,119.9,110.7$, 110.6, 99.9, 37.5, 28.0, 27.3, 22.7. IR (neat, $\left.\mathrm{cm}^{-1}\right): 3405,2937,1455,1394,749$. Anal. calcd (found) for $\mathrm{C}_{14} \mathrm{H}_{17} \mathrm{~N}$ : C, 84.37 (84.22); H, 8.60 (8.70).

For 1-Methyl-2-(4-methyl-4-pentenyl)-1 $H$-indole: $T L C$ (hexanes- $\mathrm{CH}_{2} \mathrm{Cl}_{2}=5: 1$ ): $R_{f}=$ 0.35. ${ }^{1} \mathrm{H}$ NMR: $\delta$ 7.63-7.61 (m, $\left.1 \mathrm{H}\right), 7.33$ (br d, $\left.J=7.6 \mathrm{~Hz}, 1 \mathrm{H}\right), 7.23(\mathrm{dt}, J=1.2,6.8$ 
Hz,2 H), 7.15 (dt, $J=1.0,7.6 \mathrm{~Hz}, 1 \mathrm{H}), 6.34(\mathrm{~d}, J=0.4 \mathrm{~Hz}, 1 \mathrm{H}), 4.86-4.82(\mathrm{~m}, 2 \mathrm{H}), 3.71(\mathrm{~s}, 3$ H), $2.79(\mathrm{t}, J=7.4 \mathrm{~Hz}, 2 \mathrm{H}), 2.24$ (t, $J=7.4 \mathrm{~Hz}, 2 \mathrm{H}), 1.95$ (quint, $J=7.4 \mathrm{~Hz} 2 \mathrm{H}$ ), 1.83 (s, $6 \mathrm{H}$ ). ${ }^{13} \mathrm{C}\left\{{ }^{1} \mathrm{H}\right\}$ NMR: $\delta$ 145.6, 141.4, 137.6, 128.2, 120.8, 120.0, 119.5, 110.7, 109.0, 99.1, 37.7, 29.7, 26.7, 26.6, 22.7. IR (neat, $\mathrm{cm}^{-1}$ ): 2933, 1643, 1470, 742. Anal. calcd (found) for $\mathrm{C}_{15} \mathrm{H}_{19} \mathrm{~N}$ : C, 84.46 (84.61); H, 8.98 (8.83).

2-(1,1-Dimethyl-4-pentenyl)-1-methyl-1H-indole (Table 1, entry 7). 2-(1,1-Dimethyl-4pentenyl)-1-methyl- $1 H$-indole was isolated in 34\% yield as a pale yellow oil from reaction of 2(1,1-dimethyl-4-pentenyl)-1H-indole and methyl iodide employing a procedure similar to that used to synthesize 1. TLC (hexanes- $\left.\mathrm{CH}_{2} \mathrm{Cl}_{2}=5: 1\right): R_{f}=0.36 .{ }^{1} \mathrm{H} \mathrm{NMR}: \delta$ 7.59-7.57 $(\mathrm{m}, 1 \mathrm{H})$, $7.31(\mathrm{dd}, J=0.8,8.0 \mathrm{~Hz}, 1 \mathrm{H}), 7.21(\mathrm{ddd}, J=1.2,7.6,8.0 \mathrm{~Hz}, 1 \mathrm{H}), 7.11(\mathrm{ddd}, J=1.2,6.8,7.6$ Hz, $1 \mathrm{H}), 6.35$ (d, J=0.4 Hz, $1 \mathrm{H}), 5.85-5.75(\mathrm{~m}, 1 \mathrm{H}), 5.00-4.91(\mathrm{~m}, 2 \mathrm{H}), 3.88$ (s, $3 \mathrm{H}), 1.92-$ $1.90(\mathrm{~m}, 4 \mathrm{H}), 1.51(\mathrm{~s}, 6 \mathrm{H}) .{ }^{13} \mathrm{C}\left\{{ }^{1} \mathrm{H}\right\}$ NMR: $\delta$ 147.4, 139.1, 138.7, 127.5, 121.5, 120.2, 119.6, $114.5,108.9,100.3,41.0,36.1,29.7,32.6,29.7,29.2$. IR (neat, $\left.\mathrm{cm}^{-1}\right): 2978,1469,1356,1313$, 781, 749. Anal. calcd (found) for $\mathrm{C}_{16} \mathrm{H}_{21} \mathrm{~N}: \mathrm{C}, 84.53$ (84.42); H, 9.31 (9.29).

2-(1,1-Dimethyl-4-pentenyl)-1H-indole (Table 1, entry 8). 2-(1,1-Dimethyl-4-pentenyl)$1 H$-indole was isolated in $45 \%$ yield as a pale yellow solid from reaction of $\mathbf{S 1}$ and methyl 2,2dimethyl-5-hexenoate employing a procedure similar to that used to synthesize S4. mp $42-44$ ${ }^{\circ} \mathrm{C}$. TLC (hexanes- $\left.\mathrm{CH}_{2} \mathrm{Cl}_{2}=1: 1\right): R_{f}=0.60 .{ }^{1} \mathrm{H}$ NMR: $\delta 7.93(\mathrm{~s}, 1 \mathrm{H}), 7.60-7.58(\mathrm{~m}, 1 \mathrm{H})$, 7.36-7.34 (m, $1 \mathrm{H}), 7.19-7.10(\mathrm{~m}, 2 \mathrm{H}), 6.30(\mathrm{dd}, J=1.0,2.2,1 \mathrm{H}), 5.80(\mathrm{tdd}, J=6.4,10.2,17.0$ Hz, $1 \mathrm{H})$, 5.03-4.92 (m, $2 \mathrm{H}), 1.99-1.93$ (m, $2 \mathrm{H}), 1.77-1.74$ (AA'XX', $2 \mathrm{H}), 1.42$ (s, $6 \mathrm{H})$. ${ }^{13} \mathrm{C}\left\{{ }^{1} \mathrm{H}\right\}$ NMR: $\delta$ 147.2, 139.2, 136.2, 128.8, 121.4, 120.3, 119.9, 114.5, 110.7, 98.8, 43.0, 35.3, 29.5, 28.3. IR (neat, $\mathrm{cm}^{-1}$ ): 3424, 2966, 1455, 1290, 909, 786, 750. Anal. calcd (found) for $\mathrm{C}_{15} \mathrm{H}_{19} \mathrm{~N}: \mathrm{C}, 84.46$ (84.26); H, 8.98 (9.12).

Dimethyl 2-(2-cyclohexenyl)-2-(1- methyl-1H-indol-2-ylmethyl)malonate (4, 
Table 1, entry 9). A solution of $\mathbf{S 6}(470 \mathrm{mg}, 1.71 \mathrm{mmol})$ in THF (5 mL) was added to a suspension of $\mathrm{NaH}(43 \mathrm{mg}, 1.80 \mathrm{mmol})$ in $\mathrm{THF}(15 \mathrm{~mL})$ at $0{ }^{\circ} \mathrm{C}$. The mixture was stirred for 15 min and treated with a solution of 3-chloro-1-cyclohexene $(0.39 \mathrm{~g}, 3.42 \mathrm{mmol})$ in THF $(5 \mathrm{~mL})$. The resulting mixture was refluxed for overnight and quenched with water. The layers were separated and the aqueous layer was extracted with ether $(3 \times 20 \mathrm{~mL})$. The combined organic extracts were washed (brine), dried $\left(\mathrm{MgSO}_{4}\right)$, and concentrated under vacuum. Column chromatography of the residue $\left(\mathrm{SiO}_{2}\right.$; hexanes-EtOAc = 5:1) gave compound $4(250 \mathrm{mg}, 41 \%)$ as a pale yellow oil. TLC (hexanes-EtOAc $=2: 1$ ): $R_{f}=0.60 .{ }^{1} \mathrm{H}$ NMR: $\delta 7.52(\mathrm{br} \mathrm{d}, J=7.6$ $\mathrm{Hz}, 1 \mathrm{H}), 7.26$ (br d, $J=8.0 \mathrm{~Hz}, 1 \mathrm{H}), 7.17$ (br t $, J=7.6 \mathrm{~Hz}, 1 \mathrm{H}), 7.07$ (br t, $J=7.6 \mathrm{~Hz}, 1 \mathrm{H}$ ), $6.29(\mathrm{~s}, 1 \mathrm{H}), 5.76(\mathrm{~s}, 2 \mathrm{H}), 3.68(\mathrm{~s}, 6 \mathrm{H}), 3.64(\mathrm{~s}, 3 \mathrm{H}), 3.52(\mathrm{~d}, J=15.6 \mathrm{~Hz}, 1 \mathrm{H}), 3.44(\mathrm{~d}, J=$ $16.0 \mathrm{~Hz}, 1 \mathrm{H}), 3.20-3.17$ (m, $1 \mathrm{H}), 2.00-1.97$ (m, $2 \mathrm{H}), 1.83-1.79$ (m, $2 \mathrm{H}), 1.60-1.38$ (m, $2 \mathrm{H})$. ${ }^{13} \mathrm{C}\left\{{ }^{1} \mathrm{H}\right\}$ NMR: $\delta \quad 171.4,171.1,137.4,135.8,129.4,128.1,127.9,121.3,120.4,119.7,109.3$, $101.3,61.8,52.7,52.5,40.2,30.0,29.2,25.2,24.9,22.6$. IR (neat, $\mathrm{cm}^{-1}$ ): $2948,1731,1539$, 1470, 1434, 1226. HRMS calcd (found) for $\mathrm{C}_{21} \mathrm{H}_{25} \mathrm{NO}_{4}\left(\mathrm{M}^{+}\right): 355.1784$ (355.1782).

2-(2,2-Dicarbomethoxy-4-pentenyl)-1-methyl-1H-indole (7, Table 1, entry 10). $n$-BuLi (2.5 $\mathrm{M}$ solution in hexanes, $4.2 \mathrm{~mL}, 10.5 \mathrm{mmol}$ ) was added to a solution of $\mathbf{S 2}(1.61 \mathrm{~g}, 10.0$ $\mathrm{mmol})$ in $\mathrm{THF}(10 \mathrm{~mL})$ at $-78{ }^{\circ} \mathrm{C}$. After stirring for $2 \mathrm{~min}, \mathrm{MsCl}(1.24 \mathrm{~g}, 11.0 \mathrm{mmol})$ and $\mathrm{LiBr}$ ( $2.61 \mathrm{~g}, 30.0 \mathrm{mmol})$ were added sequentially. The resulting mixture was warmed to $0{ }^{\circ} \mathrm{C}$, stirred for $30 \mathrm{~min}$, and transferred via cannula to a solution of sodium dimethyl allylmalonate [generated from dimethyl allylmalonate $(1.81 \mathrm{~g}, 10.5 \mathrm{mmol})$ and $\mathrm{NaH}(0.264 \mathrm{~g}, 11.0 \mathrm{mmol})$ in THF $(20 \mathrm{~mL})]$. The reaction mixture was stirred at room temperature for $2 \mathrm{~h}$ and quenched with water. The layers were separated and the aqueous layer was extracted with ether $(3 \times 30 \mathrm{~mL})$. The combined organic extracts were washed (brine), dried $\left(\mathrm{MgSO}_{4}\right)$, and concentrated under vacuum. Column chromatography of the residue $\left(\mathrm{SiO}_{2} ;\right.$ hexanes-EtOAc $\left.=5: 1\right)$ gave 7 
$(1.90 \mathrm{~g}, 60 \%)$ as a viscous yellow oil. TLC (hexanes-EtOAc $=2: 1): R_{f}=0.62 .{ }^{1} \mathrm{H}$ NMR: $\delta$ $7.54(\mathrm{br} \mathrm{d}, J=8.0 \mathrm{~Hz}, 1 \mathrm{H}), 7.28(\mathrm{br} \mathrm{d}, J=7.6 \mathrm{~Hz}, 1 \mathrm{H}), 7.20(\mathrm{dt}, J=1.2,8.0 \mathrm{~Hz}, 1 \mathrm{H}), 7.11-7.07$ (m, $1 \mathrm{H}), 6.27(\mathrm{~d}, J=0.8 \mathrm{~Hz}, 1 \mathrm{H}), 5.91$ (tdd, $J=7.2,10.4,17.2 \mathrm{~Hz}, 1 \mathrm{H}), 5.16-5.08(\mathrm{~m}, 2 \mathrm{H})$, $3.74(\mathrm{~s}, 6 \mathrm{H}), 3.67(\mathrm{~s}, 3 \mathrm{H}), 3.44(\mathrm{~s}, 2 \mathrm{H}), 2.81(\mathrm{~d}, J=6.8 \mathrm{~Hz}, 2 \mathrm{H}) .{ }^{13} \mathrm{C}\left\{{ }^{1} \mathrm{H}\right\}$ NMR: $\delta$ 171.4, $137.5,134.9,132.6,128.0,121.4,120.4,119.8,109.4,101.3,58.2,53.0,37.2,30.0,29.5$. IR (neat, $\mathrm{cm}^{-1}$ ): 1736, 1217, 749. Anal. calcd (found) for $\mathrm{C}_{18} \mathrm{H}_{21} \mathrm{NO}_{4}$ : C, 68.55 (68.50); $\mathrm{H}, 6.71$ (6.71).

2-(2-Carbomethoxy-4-pentenyl)-1-methyl-1H-indole (Table 1, entry 11). Compound 7 $(1.01 \mathrm{~g}, 2.7 \mathrm{mmol})$ and water $(0.3 \mathrm{~mL})$ were added sequentially to a suspension of $\mathrm{NaCl}(0.24 \mathrm{~g}$, $4.1 \mathrm{mmol})$ in DMSO $(10 \mathrm{~mL})$. The resulting mixture was refluxed for $2 \mathrm{~h}$ and then poured into brine $(20 \mathrm{~mL})$. The layers were separated and the aqueous layer was extracted with hexanes $(5 \times$ $15 \mathrm{~mL})$. The combined organic extracts were dried $\left(\mathrm{MgSO}_{4}\right)$ and concentrated under vacuum. Column chromatography of the residue $\left(\mathrm{SiO}_{2} ;\right.$ hexanes-EtOAc $\left.=10: 1\right)$ gave 2-(2carbomethoxy-4-pentenyl)-1-methyl-1 $\mathrm{H}$-indole $(0.54 \mathrm{~g}, 73 \%)$ as a pale yellow solid. $\mathrm{mp}$ 63-64 ${ }^{\circ} \mathrm{C} . \quad$ TLC (hexanes-EtOAc $\left.=2: 1\right): R_{f}=0.64 .{ }^{1} \mathrm{H}$ NMR: $\delta 7.54(\mathrm{br} \mathrm{d}, J=8.0 \mathrm{~Hz}, 1 \mathrm{H}), 7.28(\mathrm{br}$ d, $J=8.0 \mathrm{~Hz}, 1 \mathrm{H}), 7.18(\mathrm{dt}, J=1.2,8.0 \mathrm{~Hz}, 1 \mathrm{H}), 7.08(\mathrm{dt}, J=1.2,7.8 \mathrm{~Hz}, 1 \mathrm{H}), 6.29(\mathrm{~s}, 1 \mathrm{H})$, 5.79 (tdd, $J=6.8,10.4,17.2 \mathrm{~Hz}, 1 \mathrm{H}), 5.15-5.08$ (m, $2 \mathrm{H}), 3.69$ (s, $3 \mathrm{H}), 3.66$ (s, $3 \mathrm{H}), 3.18-3.11$ (m, $1 \mathrm{H}), 2.95-2.87(\mathrm{~m}, 2 \mathrm{H}), 2.53-2.37(\mathrm{~m}, 2 \mathrm{H}) .{ }^{13} \mathrm{C}\left\{{ }^{1} \mathrm{H}\right\} \mathrm{NMR}: \delta$ 175.4, 138.2, 137.7, 135.1, $128.1,121.2,120.3,119.7,117.9,109.2,100.3,52.1,45.1,36.7,29.9,28.8$. IR (neat, $\left.\mathrm{cm}^{-1}\right)$ : 2948, 1737, 1468,919, 748. HRMS calcd (found) for $\mathrm{C}_{16} \mathrm{H}_{19} \mathrm{NO}_{2}\left(\mathrm{M}^{+}\right)$: 257.1416 (257.1418).

$N$-Allyl- $N$-methyl 1-methyl-1H-indole-2-carboxamide (Table 1, entry 12). Oxallyl chloride $(3.81 \mathrm{~g}, 30.0 \mathrm{mmol})$ and DMF $(0.5 \mathrm{~mL})$ were added to a solution of 1-methyl-1Hindole-2-carboxylic acid $(1.75 \mathrm{~g}, 10.0 \mathrm{mmol})$ in $\mathrm{CH}_{2} \mathrm{Cl}_{2}(100 \mathrm{~mL})$. The resulting mixture was refluxed for $1 \mathrm{~h}$ and then concentrated under vacuum. The residue was dissolved in $\mathrm{CH}_{2} \mathrm{Cl}_{2}$ 
$(100 \mathrm{~mL})$ and treated with $N$-methyl allylamine $(2.87 \mathrm{~mL}, 30.0 \mathrm{mmol})$ at $0{ }^{\circ} \mathrm{C}$. The resulting mixture was stirred for $1 \mathrm{~h}$ at room temperature and then quenched with $1 \mathrm{~N} \mathrm{HC}$. The layers were separated and the aqueous layer was extracted with ether $(3 \times 100 \mathrm{~mL})$. The combined organic extracts were washed (brine), dried $\left(\mathrm{MgSO}_{4}\right)$, and concentrated under vacuum. Column chromatography of the residue $\left(\mathrm{SiO}_{2}\right.$; hexanes-EtOAc $\left.=3: 1\right)$ gave $N$-allyl- $N$-methyl 1-methyl$1 H$-indole-2-carboxamide $(1.92 \mathrm{~g}, 84 \%)$ as a yellow oil. TLC (hexanes-EtOAc $=2: 1): R_{f}=$ 0.32. ${ }^{1} \mathrm{H}$ NMR: $\delta 7.63(\mathrm{br} \mathrm{d}, J=8.0 \mathrm{~Hz}, 1 \mathrm{H}), 7.37(\mathrm{dd}, J=0.8,8.2 \mathrm{~Hz}, 1 \mathrm{H}), 7.30(\mathrm{dt}, J=1.0$, $7.0 \mathrm{~Hz}, 1 \mathrm{H}), 7.15$ (dt, $J=1.0,7.2 \mathrm{~Hz}, 1 \mathrm{H}), 6.69$ (br s, $1 \mathrm{H}), 5.86$ (br s, $1 \mathrm{H}), 5.35-5.28$ (m, $2 \mathrm{H})$, $4.18(\mathrm{~d}, J=2.8 \mathrm{~Hz}, 2 \mathrm{H}), 3.86(\mathrm{~s}, 3 \mathrm{H}), 3.13(\mathrm{~s}, 3 \mathrm{H}) .{ }^{13} \mathrm{C}\left\{{ }^{1} \mathrm{H}\right\} \mathrm{NMR}: \delta 164.3,138.2,133.4$, 132.1, 126.7, 123.6, 121.5, 120.4, 117.8, 110.1, 103.4, 54.2, 37.2, 31.4. IR (neat, $\left.\mathrm{cm}^{-1}\right): 3060$, 1640, 1598, 1504, 1230, 920, 748. Anal. calcd (found) for $\mathrm{C}_{14} \mathrm{H}_{16} \mathrm{~N}_{2} \mathrm{O}: \mathrm{C}, 73.66$ (73.56); H, $7.06(6.96)$.

2-(1,1-Dimethyl-3-butenyl)-1-methyl-1H-indole (Table 1, entry 14). 2-(1,1-Dimethyl-3butenyl)-1 $H$-indole (S8) was isolated in $41 \%$ yield as a yellow oil from reaction of $\mathbf{S 1}$ and methyl 2,2-dimethyl-4-pentenoate employing a procedure similar to that used to synthesize $\mathbf{S 4}$. 2-(1,1-Dimethyl-3-butenyl)-1-methyl- $1 H$-indole was isolated in 55\% yield as a yellow oil from reaction of S8 and methyl iodide employing a procedure similar to that used to synthesize $\mathbf{1}$.

For S8: $\operatorname{TLC}$ (hexanes- $\left.\mathrm{CH}_{2} \mathrm{Cl}_{2}=1: 1\right): R_{f}=0.56 .{ }^{1} \mathrm{H} \mathrm{NMR}: \delta 7.98(\mathrm{~s}, 1 \mathrm{H}), 7.60-7.57(\mathrm{~m}, 1$ H), 7.36-7.33 (m, $1 \mathrm{H}), 7.19-7.09(\mathrm{~m}, 2 \mathrm{H}), 6.30(\mathrm{dd}, J=0.8,2.0 \mathrm{~Hz}, 1 \mathrm{H}), 5.71$ (tdd, $J=6.4$, 10.2, $17.0 \mathrm{~Hz}, 1 \mathrm{H}), 5.11-5.05(\mathrm{~m}, 2 \mathrm{H}), 2.42(\mathrm{td}, J=1.0,7.6 \mathrm{~Hz}, 2 \mathrm{H}), 1.40(\mathrm{~s}, 6 \mathrm{H}) .{ }^{13} \mathrm{C}\left\{{ }^{1} \mathrm{H}\right\}$ NMR: $\delta 147.4,136.1,135.2,128.7,121.4,120.3,119.9,118.0,110.7,98.5,48.1,35.3,28.0$. IR $\left(\right.$ neat, $\mathrm{cm}^{-1}$ ): 3422, 2965, 1458, 1295, 914, 784, 749, 698. Anal. calcd (found) for $\mathrm{C}_{14} \mathrm{H}_{17} \mathrm{~N}$ : C, 84.37 (84.50); H, 8.60 (8.60).

F o r 2-(1,1-Dimethyl-3-butenyl)-1- methyl-1H-indole: $\operatorname{TLC}$ (hexanes- $\mathrm{CH}_{2} \mathrm{Cl}_{2}=$ 
5:1): $R_{f}=0.44 .{ }^{1} \mathrm{H}$ NMR: $\delta \quad 7.61-7.59(\mathrm{~m}, 1 \mathrm{H}), 7.33(\mathrm{br} \mathrm{d}, J=8.0,1 \mathrm{H}), 7.25-7.21(\mathrm{~m}, 1 \mathrm{H})$, 7.15-7.11 (m, $1 \mathrm{H}), 6.36(\mathrm{~m}, 1 \mathrm{H}), 5.64(\mathrm{tdd}, J=6.4,10.2,17.0 \mathrm{~Hz}, 1 \mathrm{H}), 5.12-5.00(\mathrm{~m}, 2 \mathrm{H})$, $3.93(\mathrm{~s}, 3 \mathrm{H}), 2.61(\mathrm{dd}, J=0.8,7.2 \mathrm{~Hz}, 2 \mathrm{H}), 1.51(\mathrm{~s}, 6 \mathrm{H}) .{ }^{13} \mathrm{C}\left\{{ }^{1} \mathrm{H}\right\} \mathrm{NMR}: \delta$ 147.6, 139.0, 135.2, 127.5, 121.2, 120.3, 119.6, 117.5, 108.9, 100.1, 46.2, 35.9, 32.7, 28.6. IR (neat, $\left.\mathrm{cm}^{-1}\right)$ : 2978, 1468, 1354, 1314, 913, 780, 749, 732. Anal. calcd (found) for $\mathrm{C}_{15} \mathrm{H}_{19} \mathrm{~N}$ : C, 84.46 (84.43); H, 8.98 (8.96).

2-(3-Butenyl)-1-methyl-1H-indole (Table 1, entry 15). 2-(3-Butenyl)-1-methyl-1H-indole was isolated in $76 \%$ yield as a yellow oil from reaction of 2-(3-butenyl)- $1 H$-indole and methyl iodide employing a procedure similar to that used to synthesize 1. TLC (hexanes- $\mathrm{CH}_{2} \mathrm{Cl}_{2}=5: 1$ ): $R_{f}=0.67 .{ }^{1} \mathrm{H}$ NMR: $\delta 7.60(\mathrm{brd}, J=7.6 \mathrm{~Hz}, 1 \mathrm{H}), 7.32(\mathrm{dd}, J=0.8,8.0 \mathrm{~Hz}, 1 \mathrm{H}), 7.22(\mathrm{ddd}, J$ $=1.2,7.6,8.0 \mathrm{~Hz}, 1 \mathrm{H}), 7.14(\mathrm{dt}, J=1.2,7.8 \mathrm{~Hz}, 1 \mathrm{H}), 6.34-6.33(\mathrm{~m}, 1 \mathrm{H}), 6.00(\mathrm{tdd}, J=6.8$, 10.4, 17.2 Hz, H), 5.23-5.10 (m, 2 H), 3.71 (s, $3 \mathrm{H}), 2.89$ (t, $J=7.6 \mathrm{~Hz}, 2 \mathrm{H}), 2.59-2.53$ (m, $2 \mathrm{H}$ ). ${ }^{13} \mathrm{C}\left\{{ }^{1} \mathrm{H}\right\}$ NMR: $\delta$ 140.8, 137.9, 137.7, 128.2, 120.9, 120.1, 119.6, 115.7, 109.0, 99.1, 32.9, 29.7, 26.7. IR (neat, $\mathrm{cm}^{-1}$ ): $3058,2928,1460,1312,933,772$. Anal. calcd (found) for $\mathrm{C}_{13} \mathrm{H}_{15} \mathrm{~N}: \mathrm{C}$, 84.28 (84.18); H, 8.16 (8.29).

\section{Dimethyl 2-(1,3-dideuterio-2-cyclohexenyl)-2-(1-methyl-1H-indol-2-ylmethyl)malonate} (4- $\left.\boldsymbol{d}_{\mathbf{2}}\right)$. Triethylamine $(12.9 \mathrm{~mL}, 90 \mathrm{mmol})$ and acetylchloride $(3.51 \mathrm{~g}, 45.0 \mathrm{mmol})$ were added sequentially to a solution of 1,3-dideuterio-2-cyclohexen-1-ol (3.00 g, $30.0 \mathrm{mmol})$ in ether (150 $\mathrm{mL})$ at $0{ }^{\circ} \mathrm{C}$. The resulting mixture was stirred at room temperature overnight and quenched with saturated aqueous $\mathrm{NH}_{4} \mathrm{Cl}$. The layers were separated and the aqueous layer was extracted with ether $(3 \times 100 \mathrm{~mL})$. The combined organic extracts were washed (brine), dried $\left(\mathrm{MgSO}_{4}\right)$, and concentrated to give 1,3-dideuterio-2-cyclohexenyl acetate $\left(\mathbf{S 9}-d_{2}\right)(3.87 \mathrm{~g}, 87 \%)$ as a colorless oil. Compound S9- $d_{2}$ was used for next step without further purification. Triphenylphosphine (371 $\mathrm{mg}, 1.42 \mathrm{mmol}), \mathrm{Pd}(\mathrm{OAc})_{2}(115 \mathrm{mg}, \quad 0.47 \mathrm{mmol})$, and $\mathbf{S}$ 9- $d_{2}(1.86 \mathrm{~g}, 11.8 \mathrm{mmol})$ 
were added sequentially to a solution of sodium dimethyl malonate [generated from dimethyl malonate $(2.34 \mathrm{~g}, 17.7 \mathrm{mmol})$ and $\mathrm{NaH}(0.45 \mathrm{~g}, 18.6 \mathrm{mmol})$ in $\mathrm{THF}(55 \mathrm{~mL})]$ at $0{ }^{\circ} \mathrm{C}$. The resulting mixture was refluxed overnight and quenched with saturated aqueous $\mathrm{NH}_{4} \mathrm{Cl}$. The layers were separated and the aqueous layer was extracted with ether $(3 \times 50 \mathrm{~mL})$. The combined organic extracts were dried $\left(\mathrm{MgSO}_{4}\right)$ and concentrated under vacuum. Column chromatography of the residue $\left(\mathrm{SiO}_{2}\right.$; hexanes-EtOAc $\left.=5: 1\right)$ gave dimethyl 2-(1,3-deuterium-2cyclohexenyl)malonate $\left(\mathbf{S 1 0}-d_{2}\right)(2.48 \mathrm{~g}, 98 \%)$ as a yellow oil. Compound $\mathbf{4}-d_{2}$ was isolated in $76 \%$ yield as a viscous yellow oil from reaction of $\mathbf{S 2}$ and $\mathbf{S 1 0}-d_{2}$ employing a procedure similar to that used to synthesize 7 .

For S9- $\boldsymbol{d}_{2}:$ TLC (hexanes-EtOAc $\left.=2: 1\right): R_{f}=0.76 .{ }^{1} \mathrm{H}$ NMR: $\delta 5.66(\mathrm{br} \mathrm{s}, 1 \mathrm{H}), 2.00(\mathrm{~s}, 3$ H), 2.00-1.57 (m, $6 \mathrm{H}) .{ }^{13} \mathrm{C}\left\{{ }^{1} \mathrm{H}\right\}$ NMR: $\delta 171.0,132.6(\mathrm{t}, J=24.1 \mathrm{~Hz}), 125.7,67.9(\mathrm{t}, J=22.8$ $\mathrm{Hz}$ ), 28.4, 25.0, 21.6, 19.0. HRMS calcd (found) for $\mathrm{C}_{8} \mathrm{H}_{10} \mathrm{D}_{2} \mathrm{O}_{2}\left(\mathrm{M}^{+}\right)$: 142.0961 (142.0965).

For S10- $\boldsymbol{d}_{2}:$ TLC (hexanes-EtOAc $\left.=2: 1\right): R_{f}=0.61 .{ }^{1} \mathrm{H}$ NMR: $\delta 5.49(\mathrm{br} \mathrm{s}, 1 \mathrm{H}), 3.71(\mathrm{~s}$, $3 \mathrm{H}), 3.70(\mathrm{~s}, 3 \mathrm{H}), 3.25$ (s, $1 \mathrm{H}), 1.97-1.94(\mathrm{~m}, 2 \mathrm{H}), 1.77-1.64(\mathrm{~m}, 2 \mathrm{H}), 1.57-1.49$ (m, $1 \mathrm{H})$, 1.37-1.30 (m, $1 \mathrm{H}) .{ }^{13} \mathrm{C}\left\{{ }^{1} \mathrm{H}\right\}$ NMR: $\delta \quad 169.1,169.1,129.7(\mathrm{t}, J=23.7 \mathrm{~Hz}), 127.4,57.0,52.6$, $35.2(\mathrm{t}, J=20.2 \mathrm{~Hz}), 26.8,25.1,21.1$. HRMS calcd (found) for $\mathrm{C}_{11} \mathrm{H}_{14} \mathrm{D}_{2} \mathrm{O}_{4}\left(\mathrm{M}^{+}\right): 214.1172$ (214.1173).

For 4- $\boldsymbol{d}_{2}$ : TLC (hexanes-EtOAc $\left.=2: 1\right): R_{f}=0.60 .{ }^{1} \mathrm{H}$ NMR: $\delta 7.52(\mathrm{br} \mathrm{d}, J=7.6 \mathrm{~Hz}, 1 \mathrm{H})$, 7.26 (br d, $J=8.0 \mathrm{~Hz}, 1 \mathrm{H}), 7.17$ (br t, $J=7.6 \mathrm{~Hz}, 1 \mathrm{H}$ ), 7.07 (br t, $J=7.6 \mathrm{~Hz}, 1 \mathrm{H}), 6.29$ (s, 1 H), 5.76 (br s, $1 \mathrm{H}), 3.69$ (s, $3 \mathrm{H}), 3.67$ (s, $3 \mathrm{H}), 3.65$ (s, $3 \mathrm{H}), 3.52$ (d, J = 16.4 Hz, $1 \mathrm{H}), 3.46$ (d, $J=15.8 \mathrm{~Hz}, 1 \mathrm{H}), 2.00-1.95(\mathrm{~m}, 2 \mathrm{H}), 1.85-1.80(\mathrm{~m}, 2 \mathrm{H}), 1.62-1.41(\mathrm{~m}, 2 \mathrm{H}) .{ }^{13} \mathrm{C}\left\{{ }^{1} \mathrm{H}\right\}$ NMR: $\delta$ $171.4,171.1,137.4,135.8,129.1(\mathrm{t}, J=23.8 \mathrm{~Hz}), 128.1,127.6,121.3,120.4,119.7,109.3$, $101.3,61.8,52.7,52.5,39.7(\mathrm{t}, J=22.0 \mathrm{~Hz}), 30.0,29.2,25.2,24.9,22.6$. HRMS calcd (found) for $\mathrm{C}_{21} \mathrm{H}_{23} \mathrm{D}_{2} \mathrm{NO}_{4}\left(\mathrm{M}^{+}\right)$: 357.1907 (355.1912). 


\section{Cyclization of 2-Alkenyl Indoles}

Table S1. Effect of Pt(II) source, catalyst loading, acid concentration, solvent, temperature, and time on the platinum-catalyzed cyclization of $\mathbf{1}$.

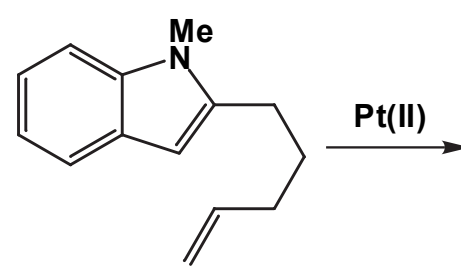

$1(0.5 \mathrm{mmol})$

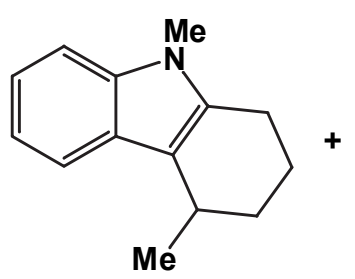

2

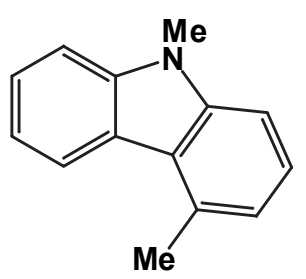

3

\begin{tabular}{clccccccc}
\hline entry & \multicolumn{1}{c}{$\mathrm{Pt}(\mathrm{II})$ source } & $\begin{array}{c}\text { cat loading } \\
(\mathrm{mol} \% \mathrm{Pt})\end{array}$ & $\begin{array}{c}\mathrm{HCl} \\
(\mathrm{mol} \%)\end{array}$ & solvent & $\begin{array}{c}\text { solvent vol. } \\
(\mathrm{mL})\end{array}$ & $\begin{array}{c}\text { temp } \\
\left({ }^{\circ} \mathrm{C}\right)\end{array}$ & $\begin{array}{c}\text { time } \\
(\mathrm{h})\end{array}$ & $\begin{array}{c}\text { product ratio } \\
(1: 2: 3)^{\mathrm{a}}\end{array}$ \\
\hline 1 & {$\left[\mathrm{PtCl}_{2}\left(\mathrm{H}_{2} \mathrm{C}=\mathrm{CH}_{2}\right)\right]_{2}$} & 5 & 0 & dioxane & 2 & 90 & 12 & $13: 67: 13$ \\
2 & {$\left[\mathrm{PtCl}_{2}\left(\mathrm{H}_{2} \mathrm{C}=\mathrm{CH}_{2}\right)\right]_{2}$} & 5 & 10 & dioxane & 2 & 90 & 12 & $<1: 83: 14$ \\
3 & {$\left[\mathrm{PtCl}_{2}\left(\mathrm{H}_{2} \mathrm{C}=\mathrm{CH}_{2}\right)\right]_{2}$} & 5 & 5 & dioxane & 2 & 90 & 12 & $<1: 85: 11$ \\
4 & {$\left[\mathrm{PtCl}_{2}\left(\mathrm{H}_{2} \mathrm{C}=\mathrm{CH}_{2}\right)\right]_{2}$} & 5 & 5 & dioxane & 2 & 60 & 24 & $<1: 85: 4$ \\
5 & {$\left[\mathrm{PtCl}_{2}\left(\mathrm{H}_{2} \mathrm{C}=\mathrm{CH}_{2}\right)\right]_{2}$} & 2 & 5 & dioxane & 1 & 60 & 24 & $<1: 92: 4$ \\
6 & $\mathrm{PtCl}_{2}$ & 2 & 5 & dioxane & 1 & 60 & 24 & $<1: 96: 2$ \\
7 & $\mathrm{PtCl}_{2}\left(\mathrm{MeCN}_{2}\right.$ & 2 & 5 & dioxane & 1 & 60 & 24 & $<1: 80: 15$ \\
8 & $\mathrm{PtCl}_{2}$ & 2 & 1 & dioxane & 1 & 60 & 24 & $<1: 90: 5$ \\
9 & $\mathrm{PtCl}_{2}$ & 2 & 5 & THF & 1 & 60 & 24 & $8: 79: 4$ \\
10 & $\mathrm{PtCl}_{2}$ & 2 & 5 & DCE & 1 & 60 & 24 & $2: 93: 3$ \\
11 & $\mathrm{PtCl}_{2}$ & 2 & 5 & toluene & 1 & 60 & 24 & $40: 57:<1$ \\
12 & none & 0 & 30 & dioxane & 1 & 60 & 24 & $92:<1:<1$ \\
\hline
\end{tabular}

${ }^{a}$ As determined by GC analysis of the crude reaction mixture.

4,9-Dimethyl-2,3,4,9-tetrahydro-1H-carbazole ( 2 ). A solution of 1-methy-2-(4pentenyl)indole (1) (97 mg, $0.50 \mathrm{mmol}), \mathrm{PtCl}_{2}(2.7 \mathrm{mg}, 0.010 \mathrm{mmol})$, and $\mathrm{HCl}$ (4.0 M solution in dioxane, $6.5 \mu \mathrm{L}, 0.025 \mathrm{mmol}$ ) was stirred at $60{ }^{\circ} \mathrm{C}$ for $18 \mathrm{~h}$, cooled to room temperature, and quenched with $1 \mathrm{~N}$ aqueous $\mathrm{NaOH}$. The layers were separated and the aqueous layer was extracted with ether $(3 \times 2 \mathrm{~mL})$. The combined organic extracts were washed (brine) 
and concentrated under vacuum. Column chromatography of the residue $\left(\mathrm{SiO}_{2}\right.$; hexanes- $\left.\mathrm{CH}_{2} \mathrm{Cl}_{2}=5: 1\right)$ gave $2(89 \mathrm{mg}, 92 \%)$ as a colorless oil. TLC (hexanes- $\left.\mathrm{CH}_{2} \mathrm{Cl}_{2}=5: 1\right): R_{f}$ $=0.37 .{ }^{1} \mathrm{H}$ NMR: $\delta 7.64(\mathrm{br} \mathrm{d}, J=7.6 \mathrm{~Hz}, 1 \mathrm{H}), 7.32-7.29(\mathrm{~m}, 1 \mathrm{H}), 7.19(\mathrm{dt}, J=1.6,7.6 \mathrm{~Hz}, 1$ H), $7.12(\mathrm{dt}, J=1.2,7.4 \mathrm{~Hz}, 1 \mathrm{H}), 3.65(\mathrm{~s}, 3 \mathrm{H}), 3.22-3.14(\mathrm{~m}, 1 \mathrm{H}), 2.72(\mathrm{dq}, J=0.8,6.2 \mathrm{~Hz}, 2$ H), 2.09-1.97 (m, $2 \mathrm{H}), 1.94-1.88(\mathrm{~m}, 2 \mathrm{H}), 1.66-1.60(\mathrm{~m}, 1 \mathrm{H}), 1.43(\mathrm{~d}, J=6.8 \mathrm{~Hz}, 3 \mathrm{H})$. ${ }^{13} \mathrm{C}\left\{{ }^{1} \mathrm{H}\right\}$ NMR: $\delta$ 137.2, 135.7, 127.0, 120.6, 118,9, 118.8, 114.5, 108.9, 32.3, 29.2, 27.5, 22.6, 21.8, 20.6. IR (neat, $\mathrm{cm}^{-1}$ ): 3051, 2927, 2857, 1470, 1375, 1313, 1244. HRMS calcd (found) for $\mathrm{C}_{14} \mathrm{H}_{17} \mathrm{~N}\left(\mathrm{M}^{+}\right): 199.1361(199.1362)$.

All remaining polycyclic indole derivatives were isolated from the corresponding 2alkenylindoles employing a similar procedure to that for the synthesis of 2 unless noted otherwise.

4,9-Dimethy-9H-carbazole (3). ${ }^{8}$ Compound 3 was isolated in $88 \%$ yield as a white solid from dehydrogenation of $2(\mathrm{Pd} / \mathrm{C})$ employing a published procedure. ${ }^{9} \quad{ }^{1} \mathrm{H}$ NMR: $\delta$ 8.30-8.27 (m, $1 \mathrm{H})$, 7.58-7.54 (m, $1 \mathrm{H})$, 7.48-7.44 (m, $1 \mathrm{H})$, 7.36-7.31 (m, $2 \mathrm{H})$, 7.12-7.10 (m, $1 \mathrm{H}), 3.86(\mathrm{~s}, 3 \mathrm{H}), 2.98(\mathrm{~s}, 3 \mathrm{H}) .{ }^{13} \mathrm{C}\left\{{ }^{1} \mathrm{H}\right\} \mathrm{NMR}: \delta 141.3,141.2,133.7,125.8,125.3,123.7$, $122.7,121.6,120.8,119.1,108.5,106.3,29.3,21.1$.

9-Benzyl-4-methyl-2,3,4,9-tetrahydro-1H-carbazole (Table 1, entry 2 ). ${ }^{10}{ }^{1} \mathrm{H}$ NMR: $\delta$ 7.73-7.69 (m, $1 \mathrm{H})$, 7.36-7.27 (m, $5 \mathrm{H})$, 7.24-7.14 (m, $2 \mathrm{H})$, 7.09-7.07 (m, $2 \mathrm{H})$, 5.30 (s, 2H), 3.31-3.23 (m, $1 \mathrm{H}), 2.70(\mathrm{dq}, J=0.8,6.2 \mathrm{~Hz}, 2 \mathrm{H}), 2.11-1.97(\mathrm{~m}, 2 \mathrm{H}), 1.93-1.86(\mathrm{~m}, 2 \mathrm{H})$, 1.73-1.65 (m, $1 \mathrm{H}), 1.50(\mathrm{~d}, J=7.2 \mathrm{~Hz}, 3 \mathrm{H}) .{ }^{13} \mathrm{C}\left\{{ }^{1} \mathrm{H}\right\} \mathrm{NMR}: \delta 138.6,137.1,135.6,129.0$, $127.5,126.5,120.9,119.1,119.0,115.1,109.4,46.5,32.2,27.5,22.6,21.8,20.6$.

6-Methoxy-4,9-dimethyl-2,3,4,9-tetrahydro-1H-carbazole (Table 1, entry 3). Yellow oil, 88\%. TLC (hexanes- $\left.\mathrm{CH}_{2} \mathrm{Cl}_{2}=1: 2\right): R_{f}=0.66 .{ }^{1} \mathrm{H} \mathrm{NMR}: \delta 7.19(\mathrm{~d}, J=8.8 \mathrm{~Hz}, 1 \mathrm{H}$ ), $7.11(\mathrm{~d}, J=2.4 \mathrm{~Hz}, 1 \mathrm{H}), 6.88(\mathrm{dd}, J=2.4, \quad 8.8 \mathrm{~Hz}, 1 \mathrm{H}), 3.93(\mathrm{~s}, 3 \mathrm{H}), 3.61(\mathrm{~s}, 3 \mathrm{H})$, 
3.21-3.13 (m, $1 \mathrm{H}), 2.78-2.64(\mathrm{~m}, 2 \mathrm{H}), 2.11-1.97(\mathrm{~m}, 3 \mathrm{H}), 1.67-1.61(\mathrm{~m}, 1 \mathrm{H}), 1.43(\mathrm{~d}, J=$ $6.8 \mathrm{~Hz}, 3 \mathrm{H}) .{ }^{13} \mathrm{C}\left\{{ }^{1} \mathrm{H}\right\}$ NMR: $\delta 153.8,136.5,132.6,127.3,114.1,110.0,109.4,101.7,56.4$, 32.2, 29.3, 27.4, 22.7, 21.5, 20.5. IR (neat, $\left.\mathrm{cm}^{-1}\right): 2930,1485,1151,1036,792$. HRMS calcd (found) for $\mathrm{C}_{15} \mathrm{H}_{19} \mathrm{NO}\left(\mathrm{M}^{+}\right)$: 229.1467 (229.1471).

2,2-Dicarbomethoxy-9-methyl-4-propyl-1,3,4,9-tetrahydrocarbazole (Table 1, entries 4 and 5). Viscous colorless oil, 80\%. TLC (hexanes-EtOAc $=2: 1): R_{f}=0.57 . \quad{ }^{1} \mathrm{H}$ NMR: $\delta 7.61($ br d, $J=8.0 \mathrm{~Hz}, 1 \mathrm{H}), 7.29(\mathrm{br} \mathrm{d}, J=8.4 \mathrm{~Hz}, 1 \mathrm{H}), 7.19(\mathrm{dt}, J=0.8,7.2 \mathrm{~Hz}, 1$ H), $7.09(\mathrm{dt}, J=0.8,7.4 \mathrm{~Hz}, 1 \mathrm{H}), 3.83(\mathrm{~s}, 3 \mathrm{H}), 3.68(\mathrm{~s}, 3 \mathrm{H}), 3.67(\mathrm{~s}, 3 \mathrm{H}), 3.51(\mathrm{~d}, J=16.0$ $\mathrm{Hz}, 1 \mathrm{H}), 3.25-3.19(\mathrm{~m}, 1 \mathrm{H}), 3.15(\mathrm{dd}, J=2.2,15.8 \mathrm{~Hz}, 1 \mathrm{H}), 2.79$ (ddd, $J=2.2,5.8,13.2$ $\mathrm{Hz}, 1 \mathrm{H}), 2.25-2.17(\mathrm{~m}, 1 \mathrm{H}), 1.95(\mathrm{dd}, J=10.4,13.2 \mathrm{~Hz}, 1 \mathrm{H}), 1.63-1.42(\mathrm{~m}, 3 \mathrm{H}), 1.01(\mathrm{t}, J$ $=7.2 \mathrm{~Hz}, 3 \mathrm{H}) .{ }^{13} \mathrm{C}\left\{{ }^{1} \mathrm{H}\right\}$ NMR: $\delta \quad 172.7,171.1,137.8,132.9,126.5,120.9,119.7,118.9$, $111.8,109.1,54.8,53.2,53.1,37.6,35.4,31.0,29.5,28.5,19.6,14.6$. IR (neat, $\mathrm{cm}^{-1}$ ): 2949 , 2871, 1730, 1469, 1380, 1236, 1236, 740. Anal. calcd (found) for $\mathrm{C}_{20} \mathrm{H}_{25} \mathrm{NO}_{4}: \mathrm{C}, 69.95$ (69.82); H, 7.34 (7.34).

4,4,9-Trimethyl-2,3,4,9-tetrahydro-1H-carbazole (Table 1, entry 6). White solid, 91\%. mp 62-64 ${ }^{\circ} \mathrm{C}$. TLC (hexanes- $\mathrm{CH}_{2} \mathrm{Cl}_{2}=5: 1$ ): $R_{f}=0.38 .{ }^{1} \mathrm{H}$ NMR: $\delta 7.73$ (br d, $J=$ $8.0 \mathrm{~Hz}, 1 \mathrm{H}), 7.31-7.28(\mathrm{~m}, 1 \mathrm{H}), 7.18(\mathrm{dt}, J=1.2,7.6 \mathrm{~Hz}, 1 \mathrm{H}), 7.10(\mathrm{dt}, J=1.2,7.4 \mathrm{~Hz}, 1$ H), $3.63(\mathrm{~s}, 3 \mathrm{H}), 2.71(\mathrm{t}, J=6.2 \mathrm{~Hz}, 2 \mathrm{H}), 2.00-1.94(\mathrm{~m}, 2 \mathrm{H}), 1.74-1.71(\mathrm{~m}, 2 \mathrm{H}), 1.49(\mathrm{~s}, 6$ H). ${ }^{13} \mathrm{C}\left\{{ }^{1} \mathrm{H}\right\}$ NMR: $\delta$ 137.3, 135.1, 126.1, 120.4, 120.1, 118.6, 117.7, 109.0, 40.7, 32.2, 29.9, 29.3, 22.8, 20.2. IR (neat, $\mathrm{cm}^{-1}$ ): 2923, 1464. Anal. calcd (found) for $\mathrm{C}_{15} \mathrm{H}_{19} \mathrm{~N}: \mathrm{C}$, 84.46 (84.43); H, 8.98 (9.12).

1,1,4,9-Tetramethyl-2,3,4,9-tetrahydro-1H-carbazole (Table 1, entry 7). Colorless oil, 91\%. TLC (hexanes- $\left.\mathrm{CH}_{2} \mathrm{Cl}_{2}=5: 1\right): R_{f}=0.40 .{ }^{1} \mathrm{H}$ NMR: $\delta$ 7.66-7.64 $(\mathrm{m}, 1 \mathrm{H}), 7.33(\mathrm{br}$ d, $J=8.0 \mathrm{~Hz}, 1 \mathrm{H}), 7.19(\mathrm{dt}, J=1.2,7.6 \mathrm{~Hz}, 1 \quad \mathrm{H}), 7.12(\mathrm{dt}, J=1.2,7.4 \mathrm{~Hz}, 1 \mathrm{H}), 3.87(\mathrm{~s}$, 
3H), 3.25-3.17 (m, $1 \mathrm{H}), 2.13-2.05(\mathrm{~m}, 1 \mathrm{H}), 2.00-1.99(\mathrm{~m}, 1 \mathrm{H}), 1.71-1.62(\mathrm{~m}, 2 \mathrm{H}), 1.54(\mathrm{~s}$, $3 \mathrm{H}), 1.46(\mathrm{~s}, 3 \mathrm{H}), 1.44(\mathrm{~d}, J=7.2 \mathrm{~Hz}, 3 \mathrm{H}) .{ }^{13} \mathrm{C}\left\{{ }^{1} \mathrm{H}\right\}$ NMR: $\delta$ 142.0, 138.0, 126.7, 121.1, $119.1,118.9,114.4,108.9,39.3,32.6,32.2,29.1,28.8,28.2,27.8,21.6 . \quad$ IR $\left(\right.$ neat, $\left.\mathrm{cm}^{-1}\right)$ : 2959, 2920, 1470, 1360, 738. Anal. calcd (found) for $\mathrm{C}_{16} \mathrm{H}_{21} \mathrm{~N}$ : C, 84.53 (84.37); H, 9.31 (9.60).

1,1,4-Trimethyl-2,3,4,9-tetrahydro-1H-carbazole (Table 1, entry 8). White solid, 87\%. mp 91-94 ${ }^{\circ} \mathrm{C} . \quad \mathrm{TLC}$ (hexanes- $\left.\mathrm{CH}_{2} \mathrm{Cl}_{2}=1: 1\right): R_{f}=0.64 .{ }^{1} \mathrm{H}$ NMR: $\delta 7.71(\mathrm{~s}, 1 \mathrm{H})$, $7.60(\mathrm{br} \mathrm{d}, J=8.0,1 \mathrm{H}), 7.337 .31(\mathrm{~m}, 1 \mathrm{H}), 7.17-7.08(\mathrm{~m}, 1 \mathrm{H}), 3.15-3.10(\mathrm{~m}, 1 \mathrm{H}), 2.10-2.03$ (m, $1 \mathrm{H}), 1.89-1.81(\mathrm{~m}, 1 \mathrm{H}), 1.69-1.59(\mathrm{~m}, 2 \mathrm{H}), 1.39(\mathrm{~d}, J=7.2 \mathrm{~Hz}, 3 \mathrm{H}), 1.36(\mathrm{~s}, 3 \mathrm{H}), 1.32$ (s, $3 \mathrm{H}) .{ }^{13} \mathrm{C}\left\{{ }^{1} \mathrm{H}\right\}$ NMR: $\delta$ 141.7, 136.2, 127.6, 121.2, 119.9, 119.3, 114.0, 110.9, 36.5, 31.8, 29.7, 29.4, 29.2, 27.7, 21.6. IR (neat, $\mathrm{cm}^{-1}$ ): 3417, 2957, 2924, 2867, 1450, 745. Anal. calcd (found) for $\mathrm{C}_{15} \mathrm{H}_{19} \mathrm{~N}$ : C, 84.46 (84.31); H, 8.98 (8.76).

\section{cis-5,5-Dicarbomethoxy-7-methyl-2,3,4a,6,7,11c-hexahydro-1H,4H-}

benzo[c]carbazole (5). White solid, $82 \%$. mp $165-166^{\circ} \mathrm{C}$. TLC (hexanes-EtOAc $\left.=2: 1\right): R_{f}$ $=0.55 .{ }^{1} \mathrm{H}$ NMR: $\delta 7.67(\mathrm{~d}, J=8.0 \mathrm{~Hz}, 1 \mathrm{H}), 7.29(\mathrm{~d}, J=8.0 \mathrm{~Hz}, 1 \mathrm{H}), 7.17(\mathrm{dt}, J=1.2,7.6$ $\mathrm{Hz}, 1 \mathrm{H}), 7.06(\mathrm{dt}, J=1.2,7.6 \mathrm{~Hz}, 1 \mathrm{H}), 3.82(\mathrm{~s}, 3 \mathrm{H}), 3.70(\mathrm{~s}, 3 \mathrm{H}), 3.68(\mathrm{~s}, 3 \mathrm{H}), 3.55(\mathrm{~d}, J=$ $17.2 \mathrm{~Hz}, 1 \mathrm{H}), 3.44-3.42$ (br s, $1 \mathrm{H}), 3.19$ (dd, $J=2.8,17.6 \mathrm{~Hz}, 1 \mathrm{H}), 2.87$ (br d, $J=14.4 \mathrm{~Hz}$, $1 \mathrm{H}), 2.83(\mathrm{td}, J=4.0,12.0,2 \mathrm{H}), 1.78(\mathrm{tt}, J=3.8,13.6 \mathrm{~Hz}, 1 \mathrm{H}), 1.66-1.63(\mathrm{~m}, 1 \mathrm{H}), 1.50-$ $1.45(\mathrm{~m}, 2 \mathrm{H}), 1.39(\mathrm{tq}, J=3.2,12.8 \mathrm{~Hz}, 1 \mathrm{H}), 1.28(\mathrm{dq}, J=4.0,12.8 \mathrm{~Hz}, 1 \mathrm{H}), 1.14(\mathrm{tq}, J=$ 3.2, $13.2 \mathrm{~Hz}, 1 \mathrm{H}) .{ }^{13} \mathrm{C}\left\{{ }^{1} \mathrm{H}\right\}$ NMR: $\delta$ 171.2, 170.8, 137.9, 133.4, 126.8, 120.7, 119.9, 119.0, 109.0, 108.9, 59.5, 53.4, 53.3, 41.3, 32.6, 29.7, 26.3, 24.6, 24.2, 21.5. IR (neat, $\mathrm{cm}^{-1}$ ): 2932 , 2854, 1742, 1469, 1237, 737. Anal. calcd (found) for $\mathrm{C}_{21} \mathrm{H}_{25} \mathrm{NO}_{4}$ : C, 70.96 (70.82); H, 7.09 (7.23). The cis stereochemistry of $\mathbf{5}$ was determined by single crystal X-ray analysis (see below). 
2,2-Dicarbomethoxy-4,9-dimethyl-1,3,4,9-tetrahydrocarbazole (8). White solid, 90\%. mp 127-129 ${ }^{\circ} \mathrm{C}$. TLC (hexanes-EtOAc $\left.=2: 1\right): R_{f}=0.54 .{ }^{1} \mathrm{H}$ NMR: $\delta 7.61(\mathrm{br} \mathrm{d}, J=$ 7.6 Hz, $1 \mathrm{H}), 7.30-7.27(\mathrm{~m}, 1 \mathrm{H}), 7.18(\mathrm{dt}, J=1.2,7.2 \mathrm{~Hz}, 1 \mathrm{H}), 7.07(\mathrm{dt}, J=1.2,7.4 \mathrm{~Hz}, 1$ H), $3.82(\mathrm{~s}, 3 \mathrm{H}), 3.68(\mathrm{~s}, 3 \mathrm{H}), 3.67(\mathrm{~s}, 3 \mathrm{H}), 3.53(\mathrm{td}, J=1.4,16.0 \mathrm{~Hz}, 1 \mathrm{H}), 3.22-3.17(\mathrm{~m}, 1$ H), $3.12(\mathrm{dd}, J=2.4,16.4 \mathrm{~Hz}, 1 \mathrm{H}), 2.72(\mathrm{ddd}, J=1.8,6.4,13.2 \mathrm{~Hz}, 1 \mathrm{H}), 1.88(\mathrm{dd}, J=10.4$, $13.6 \mathrm{~Hz}, 1 \mathrm{H}), 1.47(\mathrm{~d}, J=6.8 \mathrm{~Hz}, 3 \mathrm{H}) .{ }^{13} \mathrm{C}\left\{{ }^{1} \mathrm{H}\right\}$ NMR: $\delta 172.6,171.1,137.8,132.5,126.5$, 121.0, 119.7, 119.0, 112.8, 109.1, 54.8, 53.3, 53.1, 38.7, 29.5, 28.5, 26.4, 21.6. IR (neat, $\mathrm{cm}^{-}$ ${ }^{1}$ ): 2948, 1735, 1465, 1247, 1200, 740. Anal. calcd (found) for $\mathrm{C}_{18} \mathrm{H}_{21} \mathrm{NO}_{4}: \mathrm{C}, 68.55$ (68.57); H, 6.71 (6.73).

2-Carbomethoxy-4,9-dimethyl-1,3,4,9-tetrahydrocarbazole (Table 1, entry 11). Pale yellow oil, 98\%. TLC (hexanes-EtOAc $=2: 1$ ): $R_{f}=0.60 .{ }^{1} \mathrm{H}$ NMR (major diastereomer): $\delta$ $7.58(\mathrm{br} \mathrm{d}, J=7.6 \mathrm{~Hz}, 1 \mathrm{H}), 7.33-7.30(\mathrm{~m}, 1 \mathrm{H}), 7.20(\mathrm{dt}, J=1.2,7.2 \mathrm{~Hz}, 1 \mathrm{H}), 7.15-7.11(\mathrm{~m}$, $1 \mathrm{H}), 3.81$ (s, $3 \mathrm{H}), 3.66$ (s, $3 \mathrm{H}), 3.36-3.32(\mathrm{~m}, 1 \mathrm{H}), 3.14-2.85$ (m, $3 \mathrm{H}), 2.17-2.06(\mathrm{~m}, 2 \mathrm{H})$, $1.42(\mathrm{~d}, J=6.8 \mathrm{~Hz}, 3 \mathrm{H}) .{ }^{13} \mathrm{C}\left\{{ }^{1} \mathrm{H}\right\} \mathrm{NMR}: \delta 1763,137.5,133.5,126.5,121.1,119.8,119.0$, 113.9, 109.0, 52.5, 40.7, 36.6, 34.1, 29.2, 26.1, 25.0. IR (neat, $\mathrm{cm}^{-1}$ ): 2958, 1730, 1470, 1379, 1014, 739. HRMS calcd (found) for $\mathrm{C}_{16} \mathrm{H}_{19} \mathrm{NO}_{2}\left(\mathrm{M}^{+}\right)$: 257.1416 (257.1407).

2,4,9-Trimethyl-2,3,4,9-tetrahydro- $\beta$-1-carbolinone (Table 1, entry 12). White solid, 80\%. mp 67-69 ${ }^{\circ} \mathrm{C}$. TLC (hexanes-EtOAc $\left.=2: 1\right): R_{f}=0.32 .{ }^{1} \mathrm{H}$ NMR: $\delta 7.65(\mathrm{br} \mathrm{d}, J=$ $8.0 \mathrm{~Hz}, 1 \mathrm{H}), 7.38-7.31(\mathrm{~m}, 2 \mathrm{H}), 7.14(\mathrm{dt}, J=1.2,7.2 \mathrm{~Hz}, 1 \mathrm{H}), 4.13(\mathrm{~s}, 3 \mathrm{H}), 3.78(\mathrm{dd}, J=$ 5.0, $11.8 \mathrm{~Hz}, 1 \mathrm{H}), 3.38-3.29(\mathrm{~m}, 2 \mathrm{H}), 3.15(\mathrm{~s}, 3 \mathrm{H}), 1.41(\mathrm{~d}, J=6.8 \mathrm{~Hz}, 3 \mathrm{H}) .{ }^{13} \mathrm{C}\left\{{ }^{1} \mathrm{H}\right\}$ NMR: $\delta 162.0,139.4,125.9,124.6,123.8,123.6,120.6,120.2,110.6,57.1,34.7,31.4,27.5$, 19.0. IR (neat, $\left.\mathrm{cm}^{-1}\right)$ : 2968, 2924, 1645, 1540, 1493, 1396, 1373, 1326, 741. Anal. calcd (found) for $\mathrm{C}_{14} \mathrm{H}_{16} \mathrm{~N}_{2} \mathrm{O}: \mathrm{C}, 73.66$ (73.60); $\mathrm{H}, 7.06$ (6.96). 
solid, 82\%. mp 212-214 ${ }^{\circ} \mathrm{C}$. TLC (hexanes-EtOAc $\left.=1: 2\right): R_{f}=0.36 .{ }^{1} \mathrm{H}$ NMR: $\delta 11.12(\mathrm{~s}$, $1 \mathrm{H}), 7.67($ br d, $J=8.0 \mathrm{~Hz}, 1 \mathrm{H}), 7.60($ br d, $J=8.0 \mathrm{~Hz}, 1 \mathrm{H}), 7.32($ br t, $J=7.6 \mathrm{~Hz}, 1 \mathrm{H}$ ), 7.15 (br t, $J=7.2 \mathrm{~Hz}, 1 \mathrm{H}), 3.78$ (dd, $J=5.2,11.6 \mathrm{~Hz}, 1 \mathrm{H}), 3.45-3.34(\mathrm{~m}, 2 \mathrm{H}), 3.25(\mathrm{~s}, 3 \mathrm{H})$, $1.44(\mathrm{~d}, J=6.8 \mathrm{~Hz}, 3 \mathrm{H}) .{ }^{13} \mathrm{C}\left\{{ }^{1} \mathrm{H}\right\} \mathrm{NMR}: \delta 162.1,138.3,126.5,125.1,124.6,122.9,120.5$, 120.1, 113.3, 57.5, 34.6, 27.7, 18.9. IR (neat, $\mathrm{cm}^{-1}$ ): 3190, 1631, 1548, 1401, 1325, 780, 733. HRMS calcd (found) for $\mathrm{C}_{13} \mathrm{H}_{14} \mathrm{~N}_{2} \mathrm{O}\left(\mathrm{M}^{+}\right)$: 214.1106 (214.1102).

1,1,9-Trimethyl-2,3,4,9-tetrahydro-1H-carbazole (Table 1, entry 14). ${ }^{11}$ White solid, 89\%. TLC (hexanes- $\left.\mathrm{CH}_{2} \mathrm{Cl}_{2}=5: 1\right): R_{f}=0.40 .{ }^{1} \mathrm{H} \mathrm{NMR}: \delta 7.51(\mathrm{br} \mathrm{d}, J=8.0 \mathrm{~Hz}, 1 \mathrm{H}$ ), $7.29(\mathrm{br} \mathrm{d}, J=8.0,1 \mathrm{H}), 7.22(\mathrm{dt}, J=1.2,7.2 \mathrm{~Hz}, 1 \mathrm{H}), 7.12(\mathrm{dt}, J=1.2,7.2 \mathrm{~Hz}, 1 \mathrm{H}), 3.85$ (s, 3H), $2.76(\mathrm{t}, J=6.2 \mathrm{~Hz}, 2 \mathrm{H}), 1.92-1.88(\mathrm{~m}, 2 \mathrm{H}), 1.81-1.78(\mathrm{~m}, 2 \mathrm{H}), 1.47(\mathrm{~s}, 6 \mathrm{H})$. ${ }^{13} \mathrm{C}\left\{{ }^{1} \mathrm{H}\right\}$ NMR: $\delta \quad 142,4,137.8,127.1,121.2,119.0,118.4,109.5,108.7,43.1,32.7,32.2$, 28.7, 22.4, 20.7.

9-Methyl-2,3,4,9-tetrahydro-1H-carbazole (Table 1, entry 15). ${ }^{12}$ White solid, 74\%. ${ }^{1} \mathrm{H}$ NMR: $\delta 7.51(\mathrm{br} \mathrm{d}, J=7.6 \mathrm{~Hz}, 1 \mathrm{H}), 7.30-7.27(\mathrm{~m}, 1 \mathrm{H}), 7.19(\mathrm{dt}, J=1.2,7.6 \mathrm{~Hz}, 1 \mathrm{H})$, $7.12(\mathrm{dt}, J=1.2,7.4 \mathrm{~Hz}, 1 \mathrm{H}), 3.64(\mathrm{~s}, 3 \mathrm{H}), 2.79-2.73(\mathrm{~m}, 2 \mathrm{H}), 2.01-1.87(\mathrm{~m}, 2 \mathrm{H}) .{ }^{13} \mathrm{C}\left\{{ }^{1} \mathrm{H}\right\}$ NMR: $\delta$ 137.0, 136.0, 127.5, 120.8, 118,9, 118.0, 109.5, 108.8, 29.2, 23.6, 22.4, 21.4.

anti-5,5-Dicarbomethoxy-1,4a-dideuterio-7-methyl-2,3,4a,6,7,11c-hexahydro-1 H,4Hbenzo[c]carbazole $\left(\boldsymbol{a n t i - 5}-\boldsymbol{d}_{\mathbf{2}}\right)$. White solid, 73\%. TLC (hexanes-EtOAc $\left.=2: 1\right): \quad R_{f}=0.55$. The ${ }^{1} \mathrm{H}$ and ${ }^{13} \mathrm{C}$ resonances of anti-5- $d_{2}$ were assigned from the two-dimensional COSY (Figures S1 and S2), HMQC (Figure S3 and S4), and HMBC (Figures S5 and S6) correlation spectra and from selective decoupling of the one dimensional $800 \mathrm{MHz}{ }^{1} \mathrm{H}$ NMR spectrum. The numbering scheme for the ${ }^{1} \mathrm{H}$ and ${ }^{13} \mathrm{C}$ resonances of anti-5- $d_{2}$ are provided in Figure S7. ${ }^{1} \mathrm{H}$ NMR $(800 \mathrm{MHz}): \delta 7.69\left(\mathrm{~d}, J=8.0 \mathrm{~Hz}, 1 \mathrm{H}, H_{6}\right), 7.31\left(\mathrm{~d}, J=8.0 \mathrm{~Hz}, 1 \mathrm{H}, H_{4}\right), 7.19(\mathrm{dt}$, $\left.J=1.2,7.6 \mathrm{~Hz}, 1 \mathrm{H}, H_{5}\right), 7.08\left(\mathrm{dt}, J=1.2,7.6 \mathrm{~Hz}, 1 \mathrm{H}, H_{3}\right), 3.84\left(\mathrm{~s}, 3 \mathrm{H}, H_{22} / H_{26}\right), 3.72(\mathrm{~s}$, 
$\left.3 \mathrm{H}, H_{9}\right), 3.70\left(\mathrm{~s}, 3 \mathrm{H}, H_{26} / H_{20}\right), 3.59$ (dd, $\left.J=1.2,16.8 \mathrm{~Hz}, 1 \mathrm{H}, H_{11}\right), 3.45$ (br s, $\left.1 \mathrm{H}, H_{14}\right)$, $3.22\left(\mathrm{dd}, J=1.4,16.8 \mathrm{~Hz}, 1 \mathrm{H}, H_{11}\right), 1.78\left(\mathrm{ddd}, J=3.2,4.2,13.8 \mathrm{~Hz}, 1 \mathrm{H}, H_{15 a x}\right), 1.67$ (pt, $J=$ $\left.3.2,12.8,1 \mathrm{H}, H_{17 \text { eq }}\right), 1.53-1.48\left(\mathrm{~m}, 2 \mathrm{H}, H_{16 \mathrm{eq}} H_{18 \mathrm{eq}}\right), 1.41\left(\mathrm{tq}, J=3.6,12.8 \mathrm{~Hz}, 1 \mathrm{H}, H_{17 \mathrm{x}}\right)$, $1.29\left(\mathrm{dt}, J=4.0,12.8 \mathrm{~Hz}, 1 \mathrm{H}, H_{18 \mathrm{ax}}\right), 1.17\left(\mathrm{ddt}, J=3.2,12.8,13.8 \mathrm{~Hz}, 1 \mathrm{H}, H_{16 \mathrm{ax}}\right) .{ }^{13} \mathrm{C}\left\{{ }^{1} \mathrm{H}\right\}$ NMR $(800 \mathrm{MHz}): \delta 171.1\left(C_{19} / C_{23}\right), 170.7\left(C_{23} / C_{19}\right), 137.8\left(C_{7}\right), 133.3\left(C_{10}\right), 126.7\left(C_{2}\right)$, $120.7\left(C_{5}\right), 119.8\left(C_{6}\right), 118.9\left(C_{3}\right), 109.0\left(C_{4}\right), 108.9\left(C_{1}\right), 59.3\left(C_{12}\right), 53.3\left(C_{22} / C_{26}\right), 53.2$ $\left(C_{26} / C_{22}\right), 40.7\left(\mathrm{t}, J=20.2 \mathrm{~Hz}, C_{13}\right), 32.3\left(C_{14}\right), 29.6\left(C_{9}\right), 29.3\left(\mathrm{t}, J=21.0 \mathrm{~Hz}, C_{15}\right), 26.2\left(C_{16}\right)$, $24.6\left(C_{11}\right), 24.0\left(C_{18}\right), 21.4\left(C_{17}\right)$. 
Figure S1. COSY NMR spectrum of anti-5- $d_{2}$.
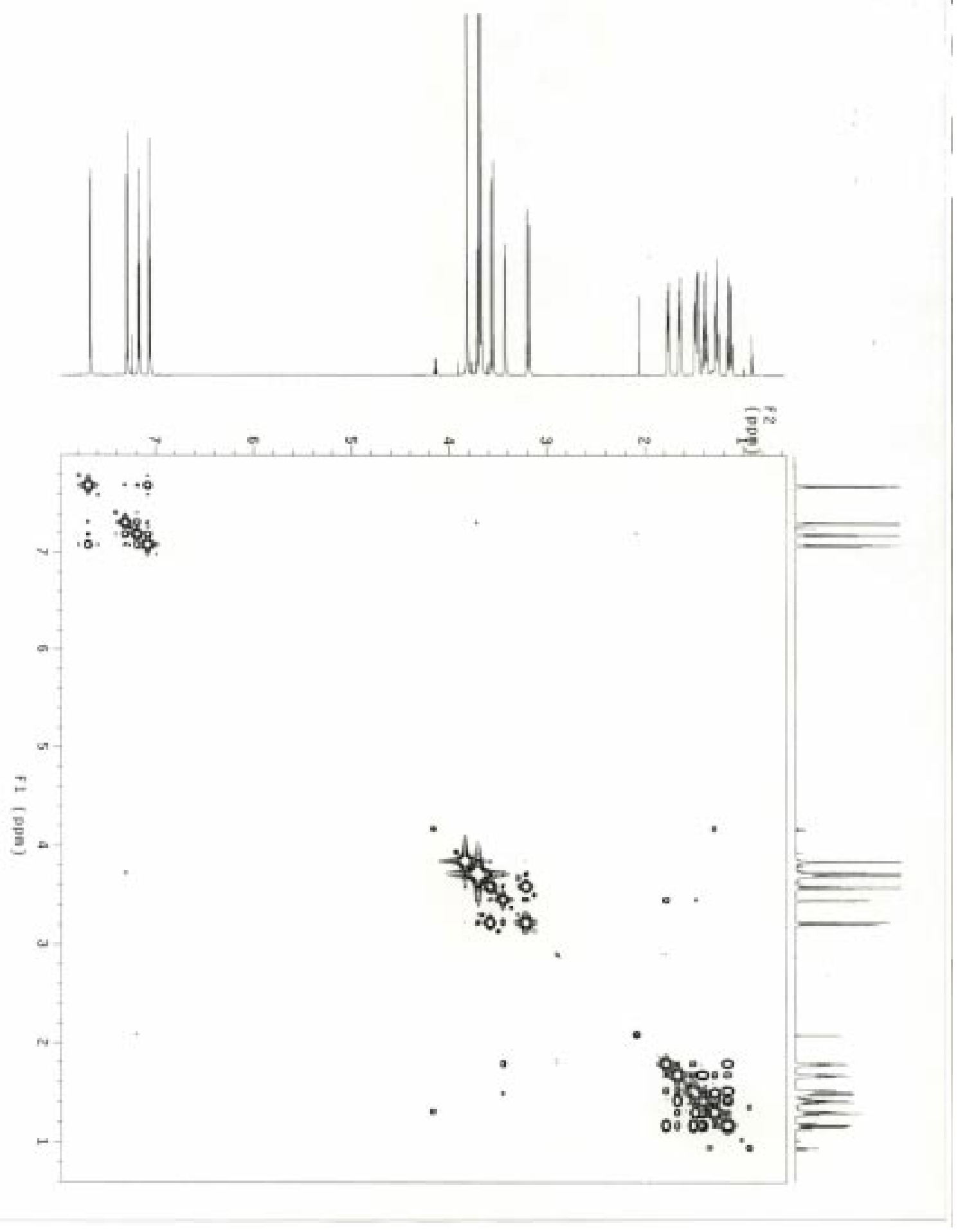
Figure S2. Partial COSY NMR spectrum of anti-5- $d_{2}$.

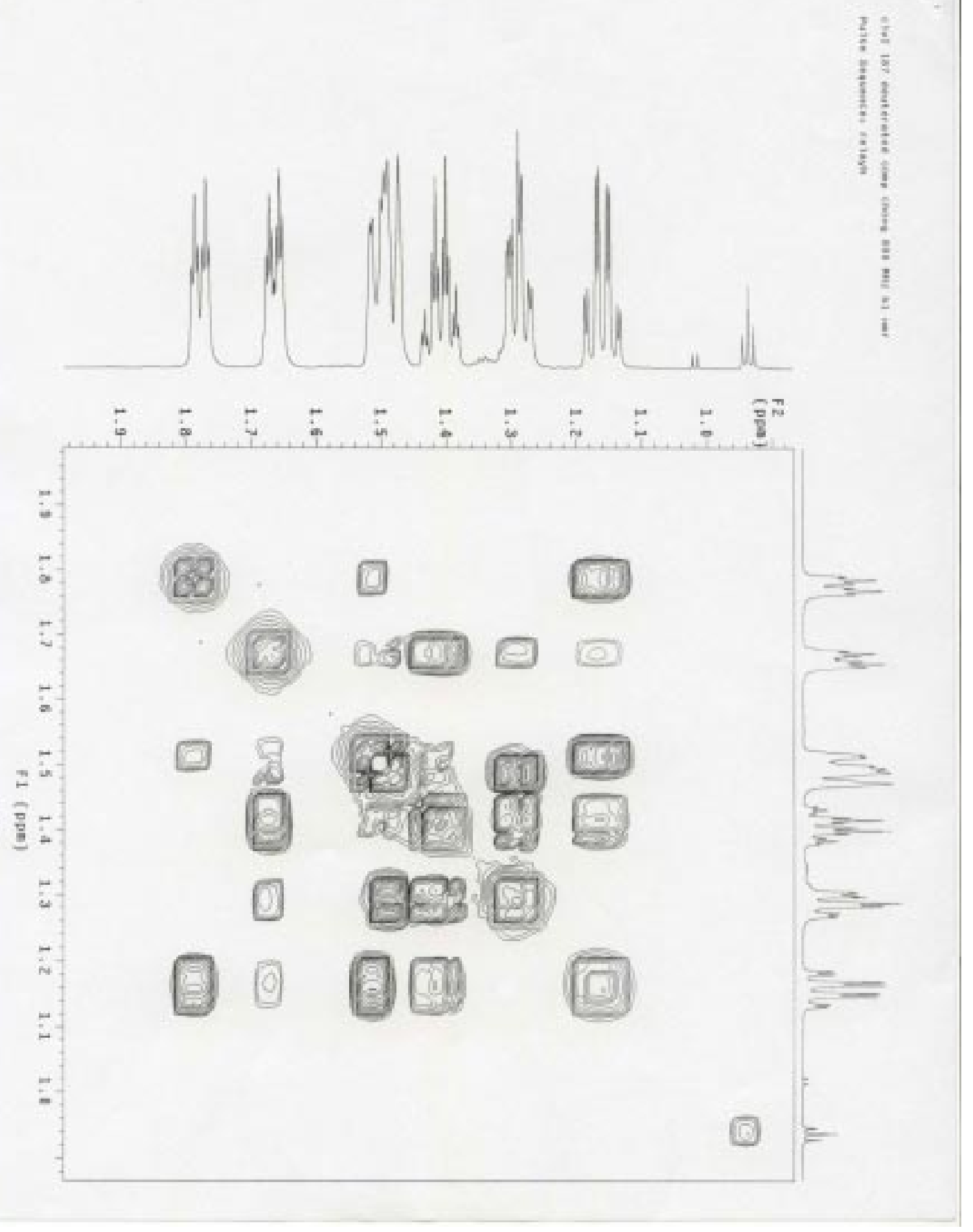


Figure S3. HMQC NMR spectrum of anti-5- $d_{2}$.

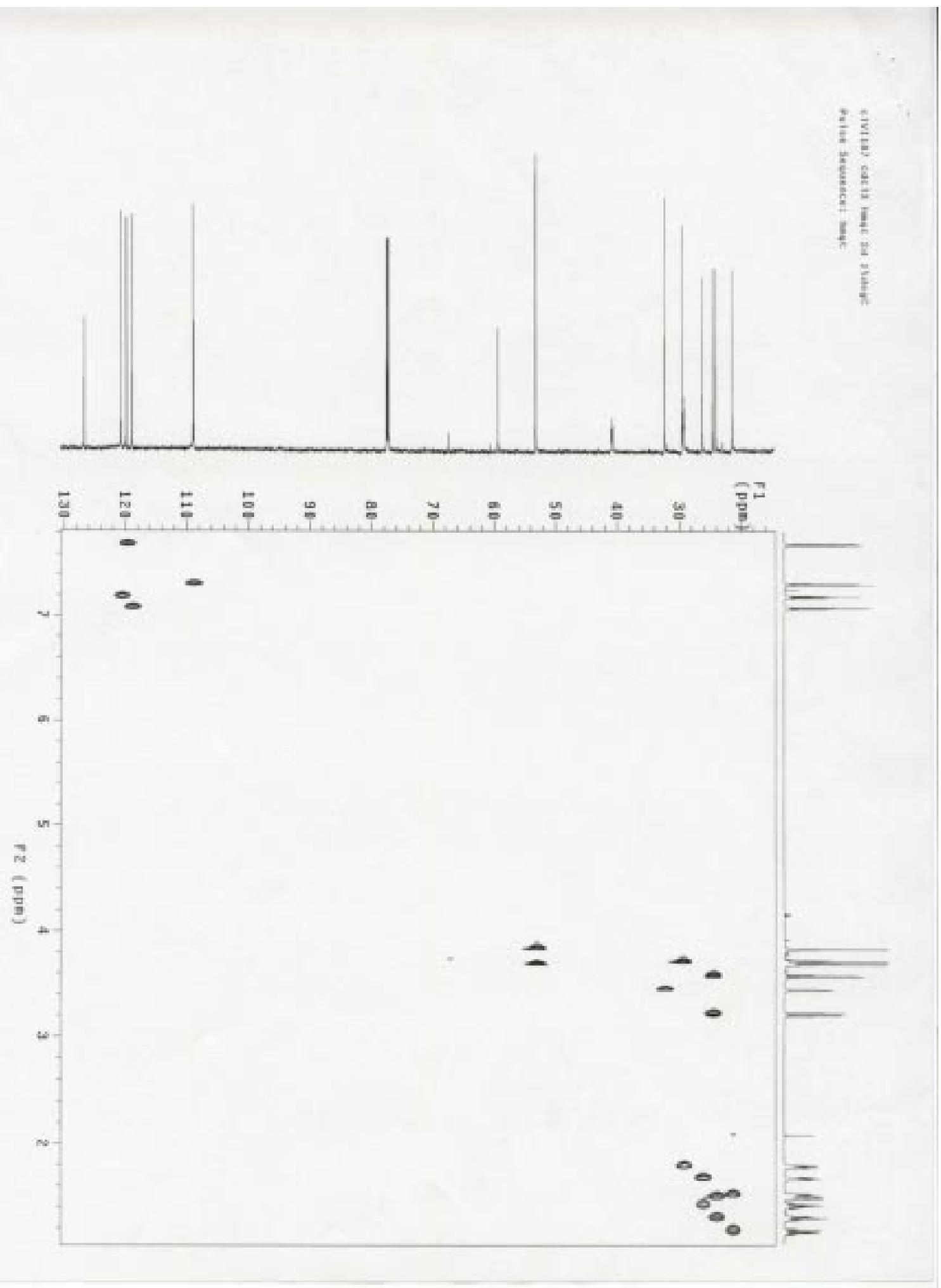


Figure S4. Partial HMQC NMR spectrum of anti-5- $d_{2}$.

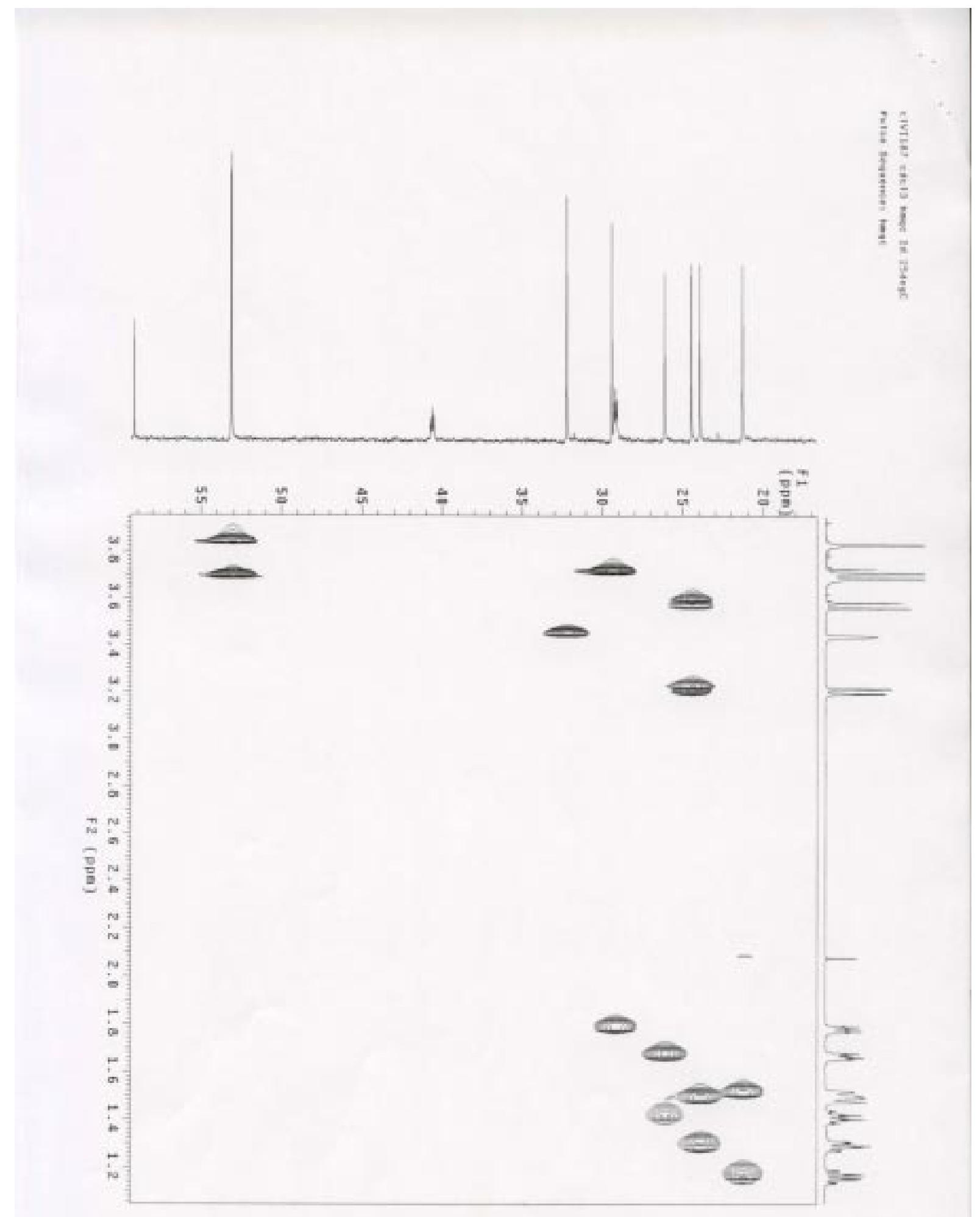

S24 
Figure S5. HMBC NMR spectrum of anti-5- $d_{2}$.

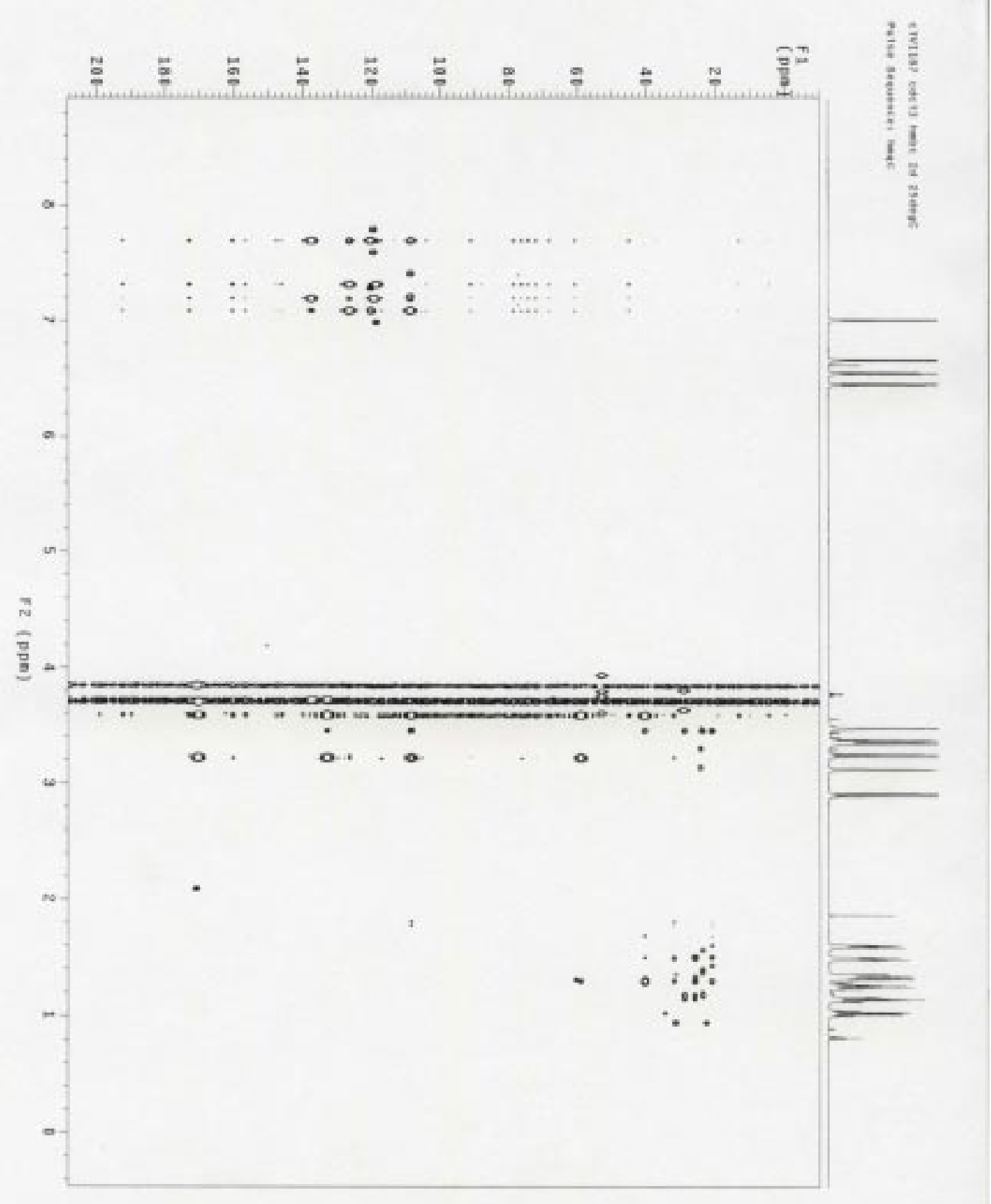


Figure S6. Partial HMBC NMR spectrum of anti-5- $d_{2}$.

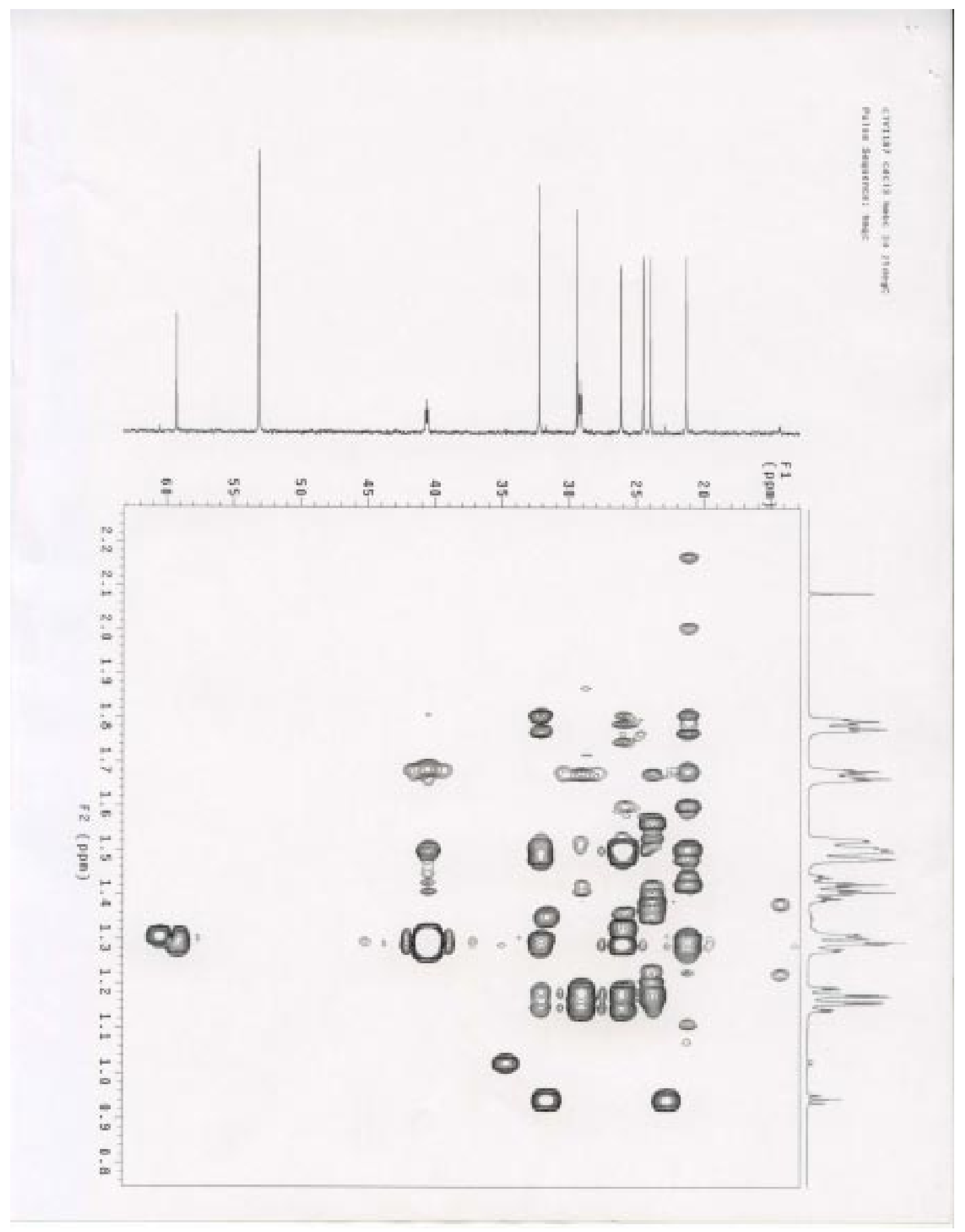


Figure S7. Numbering scheme for the ${ }^{13} \mathrm{C}$ and ${ }^{1} \mathrm{H}$ resonances of anti-5- $d_{2}$.

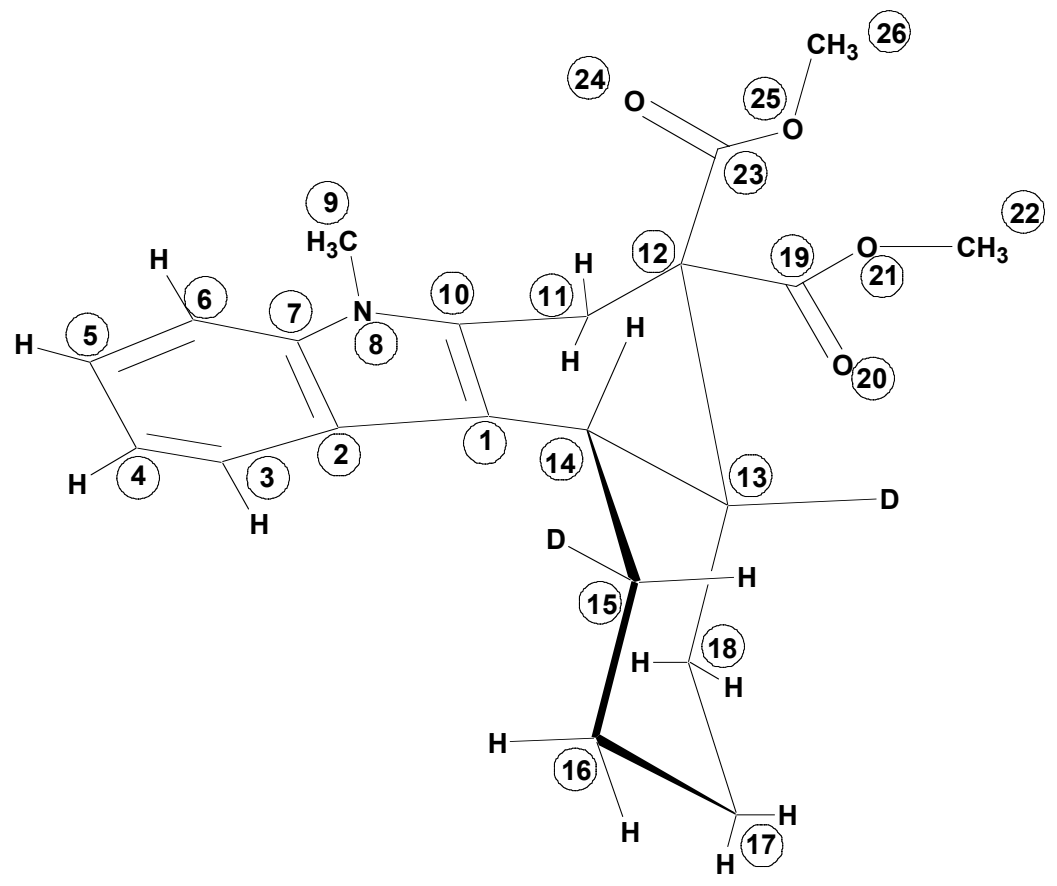

\section{Stereochemical Analysis of anti-5- $d_{2}$}

Key to the sterochemical analysis of $a n t i-5-d_{2}$ was the resonance at $\delta 1.78$ assigned to proton $H_{15 \mathrm{ax}}$ that displayed a $3.2 \mathrm{~Hz}$ coupling to $H_{16 \mathrm{eq}}$, a $4.2 \mathrm{~Hz}$ coupling to $H_{14}$ and a $13.8 \mathrm{~Hz}$ coupling to $H_{16 \mathrm{ax}}$. The large $13.8 \mathrm{~Hz}$ coupling between $H_{15 \mathrm{ax}}$ and $H_{16 \mathrm{ax}}$ is particularly diagnostic as this coupling constant establishes both the axial orientation of $H_{15 \mathrm{ax}}$ and $H_{16 \mathrm{ax}}$ and the static nature of the fused cyclohexane ring. This information, combined with the Xray crystallographic analysis of $\mathbf{5}$ establishes the anti addition of the indole and platinum across the $\mathrm{C}=\mathrm{C}$ bond of $4-d_{2}$, provided that protonolysis of the intermediate palladium alkyl intermediate anti-II- $d_{2}$ occurs with retention of stereochemistry, as has been previously established. $^{13}$ 


\section{Asymmetric Indole Alkylation}

Table S2. Effect of ligand on the yield and enantioselectivity of the conversion of $\mathbf{7}$ to $\mathbf{8}$ catalyzed by a 1:1 mixture of platinum dichloride bis(phosphine) complex (10 mol\%) and $\operatorname{AgOTf}(10 \mathrm{~mol} \%)$ in dioxane at $60{ }^{\circ} \mathrm{C}$ for $22-24 \mathrm{~h}$.

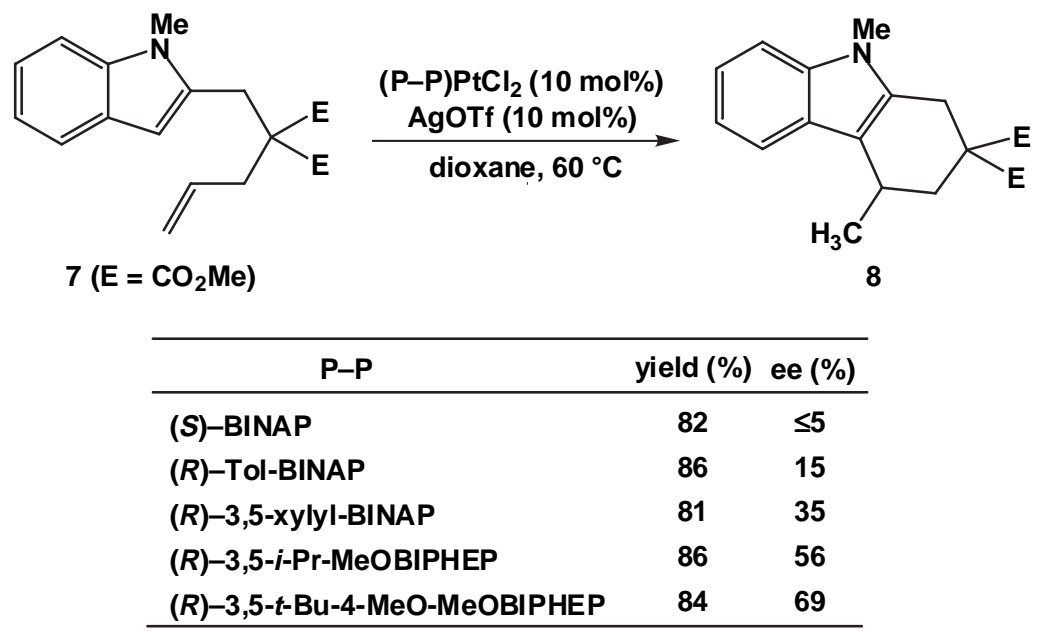

(R)-6. A mixture of $(R)-\mathbf{S 3}(89 \mathrm{mg}, 0.077 \mathrm{mmol})$ and $\mathrm{Pt}(\mathrm{COD}) \mathrm{Cl}_{2}(29 \mathrm{mg}, 0.077 \mathrm{mmol})$ in $2.5 \mathrm{~mL}$ of $\mathrm{CH}_{2} \mathrm{Cl}_{2}$ was stirred at room temperature for $20 \mathrm{~h}$. The resulting mixture was concentrated to $\sim 0.5 \mathrm{~mL}$ under vacuum and the oily residue was treated with pentane. The resulting precipitate was collected by filtration, washed by pentane, and dried under vacuum to give $(R)-6(86 \mathrm{mg}, 79 \%)$ as a pale yellow solid. ${ }^{1} \mathrm{H}$ NMR: $\delta 8.60-6.50(\mathrm{~m}, 14 \mathrm{H}), 3.68$ (s, $6 \mathrm{H}), 3.61(\mathrm{~s}, 12 \mathrm{H}), 1.37(\mathrm{~s}, 72 \mathrm{H}) .{ }^{31} \mathrm{P}\left\{{ }^{1} \mathrm{H}\right\} \mathrm{NMR} 11.4\left(\mathrm{~s},{ }^{1} J_{\mathrm{P}-\mathrm{Pt}}=3664 \mathrm{~Hz}\right)$. Anal. Calcd. (found) for $\mathrm{C}_{74} \mathrm{H}_{104} \mathrm{Cl}_{2} \mathrm{O}_{6} \mathrm{P}_{2} \mathrm{Pt}: \mathrm{C}, 62.70$ (62.56); $\mathrm{H}, 7.39$ (7.52).

Cyclization of 7 catalyzed by $(\boldsymbol{R})-6$. A suspension of 7 (87 mg, $0.28 \mathrm{mmol}),(R)-6$ (39 $\mathrm{mg}, 0.028 \mathrm{mmol})$, and $\operatorname{AgOTf}(7 \mathrm{mg}, 0.028)$ in $1.2 \mathrm{ml}$ of dioxane was stirred at $60{ }^{\circ} \mathrm{C}$ for 24 hours, cooled to room temperature, and filtered through a plug of silica gel, eluting with ether. The filtrate was concentrated under vacuum and the residue was 
chromatographed (hexanes-EtOAc $=10: 1 \rightarrow 5: 1)$ to give $8(73 \mathrm{mg}, 84 \%, 69 \%$ ee) as a white solid. The enantiomeric excess of $\mathbf{8}$ was determined by HPLC analysis using a Chiralpak AD-H column (hexanes $/ 2$-propanol $=90 / 10,0.6 \mathrm{~mL} / \mathrm{min}$ ). The retention times for the two enantiomers was 11.6 and 13.7 min, respectively (Figure S8). 
Figure S8. Chiral HPLC trace for racemic 8 (left trace) and enatiomerically enriched 8 (right trace).
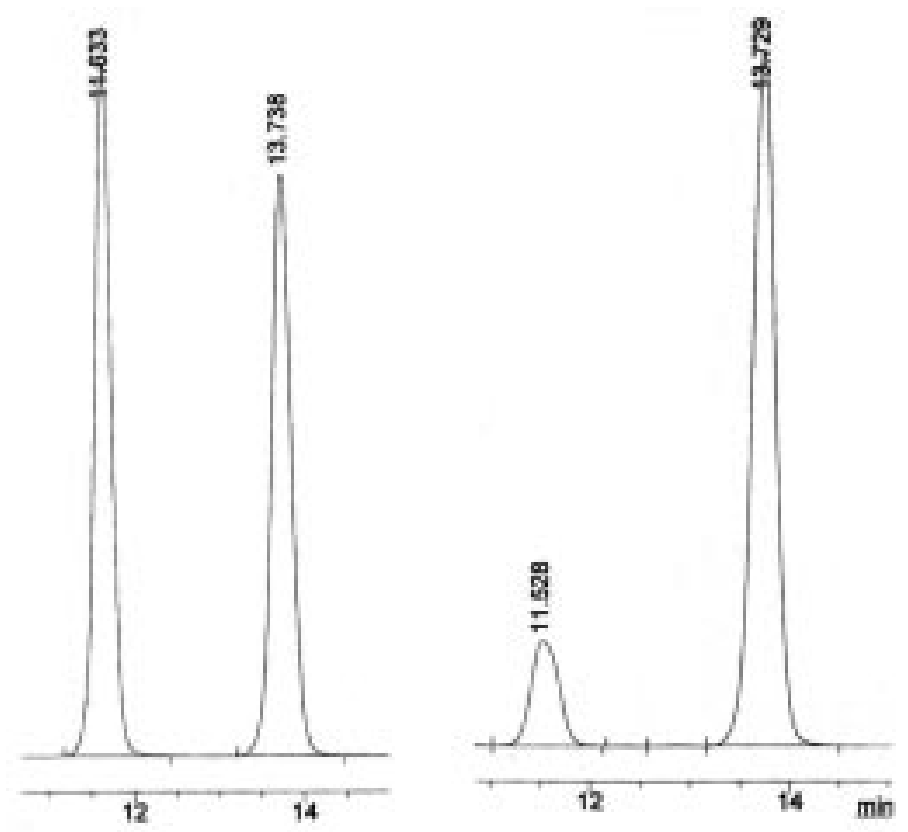


\section{X-ray Crystallography of 5.}

Crystals of $\mathbf{5}$ suitable for X-ray diffraction were obtained by slow cooling of a saturated EtOAc/hexanes $(\mathrm{v} / \mathrm{v} \approx 10 / 1)$ solution $\left(25 \rightarrow-20{ }^{\circ} \mathrm{C}\right)$. Tables of crystallographic and refinement data (Table S3), atomic coordinates (Table S4), bond lengths (Table S5), and bond angles (Table S6) are provided.

Figure S9. X-ray crystal structure of 5 .

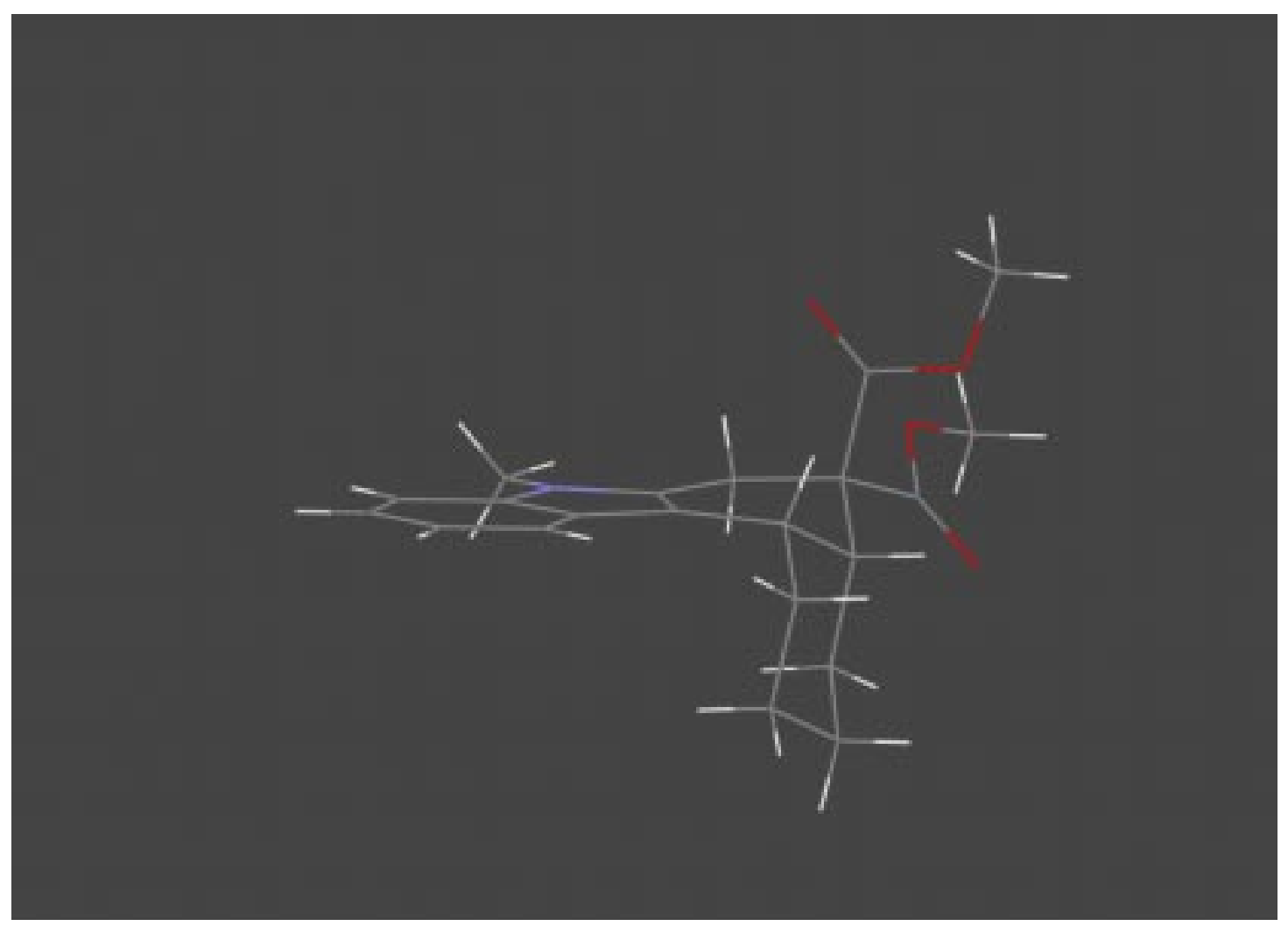


Table S3. X-ray crystal data and collection and refinement of parameters for 5.

\begin{tabular}{|c|c|}
\hline empirical formula & $\mathrm{C}_{21} \mathrm{H}_{26} \mathrm{NO}_{4}$ \\
\hline fw & 355.43 \\
\hline crystal size (mm) & $0.35 \times 0.30 \times 0.25$ \\
\hline crystal system & triclinic \\
\hline space group & $P \overline{1}$ \\
\hline$a(\AA)$ & $7.9313(2)$ \\
\hline$b(\AA)$ & $9.1118(2)$ \\
\hline$c(\AA)$ & $12.8139(3)$ \\
\hline$\alpha(\operatorname{deg})$ & $74.133(1)$ \\
\hline$\beta(\operatorname{deg})$ & $87.125(1)$ \\
\hline$\chi(\mathrm{deg})$ & 89.691(1) \\
\hline$V\left(\AA^{3}\right)$ & $889.60(4)$ \\
\hline$z$ & 2 \\
\hline scan mode & $\boldsymbol{\omega}$ \\
\hline $2 \theta$ limits (deg) & $5.00<2 \theta<56.00$ \\
\hline$D_{c}\left(\mathrm{mg}^{\star} \mathrm{cm}^{-3}\right)$ & 1.327 \\
\hline abs coeff $\left(\mathrm{mm}^{-1}\right)$ & 0.09 \\
\hline unique reflections & 4282 \\
\hline data with Inet $>2.5 \sigma(\mathrm{l})$ & 3183 \\
\hline$F(000)$ & 380.24 \\
\hline$R_{f}$ & 0.041 \\
\hline $\boldsymbol{R}_{w}$ & 0.047 \\
\hline GoF & 1.7665 \\
\hline No. of parameters & 336 \\
\hline $\max \Delta / \sigma$ & 0.000 \\
\hline largest resid density $\left(e / \AA^{3}\right)$ & 0.340 \\
\hline
\end{tabular}


Table S4. Atomic coordinates for 5.

\begin{tabular}{|c|c|c|c|c|}
\hline & $\mathrm{x}$ & $\mathrm{y}$ & $\mathrm{z}$ & $\mathrm{Biso}^{\mathrm{a}}$ \\
\hline $\mathrm{C} 1$ & $0.89147(17)$ & $0.34476(17)$ & $0.76227(11)$ & $1.45(6)$ \\
\hline $\mathrm{C} 2$ & $1.02897(17)$ & $0.45464(17)$ & $0.73001(12)$ & $1.47(6)$ \\
\hline C3 & $1.18609(18)$ & $0.47509(19)$ & $0.77092(13)$ & $1.71(7)$ \\
\hline $\mathrm{C} 4$ & $1.28828(19)$ & $0.59665(19)$ & $0.71567(13)$ & $2.04(7)$ \\
\hline C5 & $1.24003(20)$ & $0.70027(20)$ & $0.61963(14)$ & $2.07(7)$ \\
\hline C6 & $1.08661(19)$ & $0.68487(19)$ & $0.57668(13)$ & $1.88(6)$ \\
\hline C7 & $0.98335(18)$ & $0.56221(17)$ & $0.63266(12)$ & $1.55(6)$ \\
\hline N8 & $0.82731(15)$ & $0.51992(14)$ & $0.60653(10)$ & $1.55(5)$ \\
\hline C9 & $0.73063(22)$ & $0.60632(22)$ & $0.51694(15)$ & $2.09(7)$ \\
\hline $\mathrm{C} 10$ & $0.77537(18)$ & $0.38893(17)$ & $0.68455(12)$ & $1.45(6)$ \\
\hline C11 & $0.61236(18)$ & $0.31016(18)$ & $0.67917(13)$ & $1.52(6)$ \\
\hline $\mathrm{C} 12$ & $0.59723(17)$ & $0.15900(17)$ & $0.76979(12)$ & $1.43(6)$ \\
\hline C13 & $0.66811(17)$ & $0.17500(18)$ & $0.87755(12)$ & $1.42(6)$ \\
\hline C14 & $0.86133(17)$ & $0.20713(17)$ & $0.85946(12)$ & $1.43(6)$ \\
\hline C15 & $0.93319(19)$ & $0.22176(19)$ & $0.96568(12)$ & $1.73(6)$ \\
\hline C16 & $0.84644(20)$ & $0.34656(20)$ & $1.00644(14)$ & $1.94(7)$ \\
\hline $\mathrm{C} 17$ & $0.65616(19)$ & $0.31652(21)$ & $1.02314(13)$ & $1.86(6)$ \\
\hline $\mathrm{C} 18$ & $0.57863(19)$ & $0.29935(19)$ & ) $0.92023(13)$ & $1.66(7)$ \\
\hline C19 & $0.41154(18)$ & $0.10760(17)$ & $0.78372(12)$ & $1.57(6)$ \\
\hline $\mathrm{O} 20$ & $0.32050(13)$ & $0.08882(13)$ & ) $0.86410(9)$ & $2.22(5)$ \\
\hline $\mathrm{O} 21$ & $0.36117(12)$ & $0.08697(13)$ & $0.68997(9)$ & $2.05(5)$ \\
\hline $\mathrm{C} 22$ & $0.18537(20)$ & $0.04301(23)$ & $0.69048(16)$ & $2.29(8)$ \\
\hline $\mathrm{C} 23$ & $0.69535(17)$ & $0.03326(18)$ & ) $0.73374(12)$ & $1.59(6)$ \\
\hline $\mathrm{O} 24$ & $0.78018(14)$ & $0.05045(13)$ & $0.65110(9)$ & $2.47(5)$ \\
\hline $\mathrm{O} 25$ & $0.67282(13)$ & $-0.10075(12)$ & $0.80911(9)$ & $2.16(5)$ \\
\hline C26 & $0.7582(3)$ & $-0.22989(22)$ & $0.78582(19)$ & $3.00(9)$ \\
\hline H3 & $1.2228(18)$ & $0.4059(18)$ & $0.8369(13)$ & $1.3(3)$ \\
\hline H4 & $1.3938(22)$ & $0.6099(20)$ & $0.7455(14)$ & 2.7 (4) \\
\hline H5 & $1.3151(20)$ & 0.7855 (19) & $0.5817(13)$ & $2.0(3)$ \\
\hline H6 & $1.0527(21)$ & $0.7539(20)$ & $0.5100(14) 2$ & $2.3(4)$ \\
\hline H9a & 0.788 & 0.623 (3) 0. & 6.4477 (21) 6.7 & 7 (7) \\
\hline $\mathrm{H} 9 \mathrm{~b}$ & 0.633 (4) & 0.550 (3) 0. & 9.5099 (24) 9.4 & $4(9)$ \\
\hline $\mathrm{H} 9 \mathrm{c}$ & $0.701 \quad(3)$ & 0.700 (3) 0. & .5289 (21) 7.5 & 5 (7) \\
\hline H11a & 0.6074 (19) & 0.2860 & 0.6110 & $1.9(3)$ \\
\hline $\mathrm{H} 11 \mathrm{~b}$ & $0.5168(19)$ & $0.3757(18)$ & 0.6848 & $1.6(3)$ \\
\hline H13 & $0.6475(17)$ & $0.0787(18)$ & $0.9321(12)$ & $1.0(3)$ \\
\hline H14 & 0.9169 (17) & 0.1109 (17) & 0.8459 & $1.0(3)$ \\
\hline H15a & 0.9159 (19) & 0.1225 & 1.0220 & $1.8(3)$ \\
\hline $\mathrm{H} 15 \mathrm{~b}$ & $1.0589(21)$ & $0.2386(18)$ & 0.9554 & $2.1(3)$ \\
\hline H16a & 0.8675 (19) & 0.4471 (19) & 0.9503 & $1.7(3)$ \\
\hline H16b & $0.8973(20)$ & $0.3514(18)$ & 1.0768 & $2.1(3)$ \\
\hline $\mathrm{H} 17 \mathrm{a}$ & 0.6347 (19) & 0.2210 (19) & 1.0817 & $1.9(3)$ \\
\hline $\mathrm{H} 17 \mathrm{~b}$ & $0.6001(20)$ & $0.3995(20)$ & 1.0490 & $2.2(3)$ \\
\hline H18a & $0.5866(20)$ & 0.3975 & 0.8649 & $1.9(3)$ \\
\hline $\mathrm{H} 18 \mathrm{~b}$ & $0.4572(20)$ & $0.2753(18)$ & 0.93 & 353 (13) \\
\hline
\end{tabular}


Table S4. Continued

$$
\begin{array}{lllll}
\mathrm{H} 22 \mathrm{a} & 0.1727(21) & 0.0292(21) & 0.6176(15) & 3.0(4) \\
\mathrm{H} 22 \mathrm{~b} & 0.1632(21) & -0.0528(22) & 0.7486(15) & 2.8(4) \\
\mathrm{H} 22 \mathrm{c} & 0.1130(22) & 0.1229(21) & 0.6998(14) & 2.4(4) \\
\mathrm{H} 26 \mathrm{a} & 0.717(3) & -0.245(3) & 0.7130(20) & 6.1(6) \\
\mathrm{H} 26 \mathrm{~b} & 0.724(3) & -0.318(3) & 0.8408(19) & 5.3(6) \\
\mathrm{H} 26 \mathrm{c} & 0.876(3) & -0.212(3) & 0.7752(17) & 4.9(5)
\end{array}
$$

${ }^{\text {a }}$ Biso is the Mean of the Principal Axes of the Thermal Ellpsoid. 
Table S5. Bond lengths for 5 .

$\begin{array}{llll}\mathrm{C}(1)-\mathrm{C}(2) & 1.4501(20) & \mathrm{C}(14)-\mathrm{C}(15) & 1.5405(21) \\ \mathrm{C}(1)-\mathrm{C}(10) & 1.3677(20) & \mathrm{C}(14)-\mathrm{H}(14) & 1.032(15) \\ \mathrm{C}(1)-\mathrm{C}(14) & 1.5167(21) & \mathrm{C}(15)-\mathrm{C}(16) & 1.5230(22) \\ \mathrm{C}(2)-\mathrm{C}(3) & 1.4085(20) & \mathrm{C}(15)-\mathrm{H}(15 \mathrm{a}) & 0.996(17) \\ \mathrm{C}(2)-\mathrm{C}(7) & 1.4215(21) & \mathrm{C}(15)-\mathrm{H}(15 \mathrm{~b}) & 1.006(16) \\ \mathrm{C}(3)-\mathrm{C}(4) & 1.3814(23) & \mathrm{C}(16)-\mathrm{C}(17) & 1.5292(22) \\ \mathrm{C}(3)-\mathrm{H}(3) & 0.964(16) & \mathrm{C}(16)-\mathrm{H}(16 \mathrm{a}) & 1.007(17) \\ \mathrm{C}(4)-\mathrm{C}(5) & 1.4001(25) & \mathrm{C}(16)-\mathrm{H}(16 \mathrm{~b}) & 1.018(17) \\ \mathrm{C}(4)-\mathrm{H}(4) & 0.959(18) & \mathrm{C}(17)-\mathrm{C}(18) & 1.5286(22) \\ \mathrm{C}(5)-\mathrm{C}(6) & 1.3836(23) & \mathrm{C}(17)-\mathrm{H}(17 \mathrm{a}) & 0.991(18) \\ \mathrm{C}(5)-\mathrm{H}(5) & 0.982(17) & \mathrm{C}(17)-\mathrm{H}(17 \mathrm{~b}) & 0.999(18) \\ \mathrm{C}(6)-\mathrm{C}(7) & 1.3953(22) & \mathrm{C}(18)-\mathrm{H}(18 \mathrm{a}) & 0.978(18) \\ \mathrm{C}(6)-\mathrm{H}(6) & 0.962(18) & \mathrm{C}(18)-\mathrm{H}(18 \mathrm{~b}) & 0.987(16) \\ \mathrm{C}(7)-\mathrm{N}(8) & 1.3818(18) & \mathrm{C}(19)-\mathrm{O}(20) & 1.2007(18) \\ \mathrm{N}(8)-\mathrm{C}(9) & 1.4538(20) & \mathrm{C}(19)-\mathrm{O}(21) & 1.3456(18) \\ \mathrm{N}(8)-\mathrm{C}(10) & 1.3803(19) & \mathrm{O}(21)-\mathrm{C}(22) & 1.4520(18) \\ \mathrm{C}(9)-\mathrm{H}(9 \mathrm{a}) & 0.95(3) & \mathrm{C}(22)-\mathrm{H}(22 \mathrm{a}) & 0.986(19) \\ \mathrm{C}(9)-\mathrm{H}(9 \mathrm{~b}) & 0.96(3) & \mathrm{C}(22)-\mathrm{H}(22 \mathrm{~b}) & 0.990(20) \\ \mathrm{C}(9)-\mathrm{H}(9 \mathrm{c}) & 0.93(3) & \mathrm{C}(22)-\mathrm{H}(22 \mathrm{c}) & 0.953(19) \\ \mathrm{C}(10)-\mathrm{C}(11) & 1.4957(20) & \mathrm{C}(23)-\mathrm{O}(24) & 1.1989(18) \\ \mathrm{C}(11)-\mathrm{C}(12) & 1.5404(21) & \mathrm{C}(23)-\mathrm{O}(25) & 1.3401(19) \\ \mathrm{C}(11)-\mathrm{H}(11 \mathrm{a}) & 0.960(17) & \mathrm{O}(25)-\mathrm{C}(26) & 1.4475(20) \\ \mathrm{C}(11)-\mathrm{H}(11 \mathrm{~b}) & 0.974(16) & \mathrm{C}(26)-\mathrm{H}(26 \mathrm{a}) & 1.047(24) \\ \mathrm{C}(12)-\mathrm{C}(13) & 1.5598(20) & \mathrm{C}(26)-\mathrm{H}(26 \mathrm{~b}) & 0.94(3) \\ \mathrm{C}(12)-\mathrm{C}(19) & 1.5348(20) & \mathrm{C}(26)-\mathrm{H}(26 \mathrm{c}) & 0.949(23) \\ \mathrm{C}(12)-\mathrm{C}(23) & 1.5412(20) & \mathrm{H}(9 \mathrm{a})-\mathrm{H}(9 \mathrm{~b}) & 1.49(4) \\ \mathrm{C}(13)-\mathrm{C}(14) & 1.5556(20) & \mathrm{H}(9 \mathrm{a})-\mathrm{H}(9 \mathrm{c}) & 1.54(4) \\ \mathrm{C}(13)-\mathrm{C}(18) & 1.5420(21) & \mathrm{H}(11 \mathrm{a})-\mathrm{H}(11 \mathrm{~b}) & 1.557(23) \\ \mathrm{C}(13)-\mathrm{H}(13) & 0.969(16) & & \end{array}$


Table S6. Bond angles for 5 .

\begin{tabular}{|c|c|c|}
\hline$C(2)-C(1)-C(10)$ & 105.97(13) & $C(13)-C(14)-C(15)$ \\
\hline$C(2)-C(1)-C(14)$ & $132.44(12)$ & $\mathrm{C}(13)-\mathrm{C}(14)-\mathrm{H}(14)$ \\
\hline $\mathrm{C}(10)-\mathrm{C}(1)-\mathrm{C}(14)$ & $121.58(13)$ & $\mathrm{C}(15)-\mathrm{C}(14)-\mathrm{H}(14) \quad 105.0(8)$ \\
\hline $\mathrm{C}(1)-\mathrm{C}(2)-\mathrm{C}(3) \quad 1$ & 135.71(14) & $\mathrm{C}(14)-\mathrm{C}(15)-\mathrm{C}(16) \quad 112.12(12)$ \\
\hline$C(1)-C(2)-C(7)$ & $106.73(12)$ & $\mathrm{C}(14)-\mathrm{C}(15)-\mathrm{H}(15 \mathrm{a}) \quad 108.3(9)$ \\
\hline$C(3)-C(2)-C(7)$ & $117.56(14)$ & $\mathrm{C}(14)-\mathrm{C}(15)-\mathrm{H}(15 \mathrm{~b}) \quad 109.9(9)$ \\
\hline $\mathrm{C}(2)-\mathrm{C}(3)-\mathrm{C}(4)$ & $119.34(15)$ & $\mathrm{C}(16)-\mathrm{C}(15)-\mathrm{H}(15 \mathrm{a}) \quad 108.8(9)$ \\
\hline $\mathrm{C}(2)-\mathrm{C}(3)-\mathrm{H}(3)$ & $120.9(9)$ & $\mathrm{C}(16)-\mathrm{C}(15)-\mathrm{H}(15 \mathrm{~b}) \quad 111.6(9)$ \\
\hline $\mathrm{C}(4)-\mathrm{C}(3)-\mathrm{H}(3)$ & $119.7(9)$ & $\mathrm{H}(15 \mathrm{a})-\mathrm{C}(15)-\mathrm{H}(15 \mathrm{~b}) 106.0(13)$ \\
\hline$C(3)-C(4)-C(5)$ & $121.72(14)$ & $\mathrm{C}(15)-\mathrm{C}(16)-\mathrm{C}(17) \quad 110.38(13)$ \\
\hline $\mathrm{C}(3)-\mathrm{C}(4)-\mathrm{H}(4)$ & 118.1(10) & $\mathrm{C}(15)-\mathrm{C}(16)-\mathrm{H}(16 \mathrm{a}) \quad 108.5(9)$ \\
\hline $\mathrm{C}(5)-\mathrm{C}(4)-\mathrm{H}(4)$ & & $\mathrm{C}(15)-\mathrm{C}(16)-\mathrm{H}(16 \mathrm{~b}) 109$. \\
\hline$C(4)-C(5)-C(6)$ & $120.92(15)$ & $\mathrm{C}(17)-\mathrm{C}(16)-\mathrm{H}(16 \mathrm{a}) \quad 109.0(8)$ \\
\hline $\mathrm{C}(4)-\mathrm{C}(5)-\mathrm{H}(5)$ & $120.2(9)$ & $\mathrm{C}(17)-\mathrm{C}(16)-\mathrm{H}(16 \mathrm{~b}) \quad 111.0(9)$ \\
\hline $\mathrm{C}(6)-\mathrm{C}(5)-\mathrm{H}(5)$ & 118.9 & $\mathrm{H}(16 \mathrm{a})-\mathrm{C}(16)-\mathrm{H}(16 \mathrm{~b}) 108.7(12)$ \\
\hline (7) & 117.3 & (18) 111.9 \\
\hline & & 7a) 109 \\
\hline $\mathrm{C}(7)-\mathrm{C}(6)-\mathrm{H}(6)$ & 120.8 & $\mathrm{C}(16)-\mathrm{C}(17)-\mathrm{H}(17 \mathrm{~b}) \quad 109.8(9)$ \\
\hline$C(2)-C(7)-C(6)$ & 123.14( & $\mathrm{C}(18)-\mathrm{C}(17)-\mathrm{H}(17 \mathrm{a}) \quad 108.3(9)$ \\
\hline$-\mathrm{N}(8)$ & 108 & $\mathrm{C}(17)-\mathrm{H}(17 \mathrm{~b}) \quad 111.2(9)$ \\
\hline $\mathrm{C}(6$ & 128 & $\mathrm{H}(17 \mathrm{a})-\mathrm{C}(17)-\mathrm{H}(17 \mathrm{~b}) 106$ \\
\hline $\mathrm{C}(7)-\mathrm{N}(8)-\mathrm{C}(9)$ & $125.25(1$ & $\mathrm{C}(13)-\mathrm{C}(18)-\mathrm{C}(17) \quad 111.58(12)$ \\
\hline $\mathrm{C}(7)-\mathrm{N}(8)-\mathrm{C}(10)$ & 108.11(12) & $\mathrm{C}(13)-\mathrm{C}(18)-\mathrm{H}(18 \mathrm{a}) \quad 110.1(9)$ \\
\hline $\mathrm{C}(9)-\mathrm{N}(8)-\mathrm{C}(10)$ & 126. & $\mathrm{C}(13)-\mathrm{C}(18)-\mathrm{H}(18 \mathrm{~b}) \quad 110.4(9)$ \\
\hline$)-\mathrm{H}(9 \mathrm{a})$ & & $\mathrm{C}(17)-\mathrm{C}(18)-\mathrm{H}(18 \mathrm{a}) \quad 108.7(9)$ \\
\hline & 110 & $\mathrm{C}(17)-\mathrm{C}(18)-\mathrm{H}(18 \mathrm{~b}) \quad 109.4(9)$ \\
\hline $\mathrm{N}(8)-\mathrm{C}(9)-\mathrm{H}(9 \mathrm{c})$ & $109.0(16)$ & $\mathrm{H}(18 \mathrm{a})-\mathrm{C}(18)-\mathrm{H}(18 \mathrm{~b}) 106.5(13)$ \\
\hline $\mathrm{H}(9 \mathrm{a})-\mathrm{C}(9)-\mathrm{H}(9 \mathrm{~b})$ & $102.8(22)$ & $\mathrm{C}(12)-\mathrm{C}(19)-\mathrm{O}(20) \quad 126.95(13)$ \\
\hline $\mathrm{H}(9 \mathrm{a})-\mathrm{C}(9)-\mathrm{H}(9 \mathrm{c})$ & & $\mathrm{C}(12)-\mathrm{C}(19)-\mathrm{O}(21) \quad 109.64(12)$ \\
\hline 9) $-\mathrm{H}(9 \mathrm{c})$ & 111. & $\mathrm{O}(20)-\mathrm{C}(19)-\mathrm{O}(21)$ \\
\hline $\mathrm{C}(1)-\mathrm{C}(10)-\mathrm{N}(8)$ & $111.18(13)$ & $\mathrm{C}(19)-\mathrm{O}(21)-\mathrm{C}(22)$ \\
\hline$C(1)-C(10)-C(11)$ & $126.84(14)$ & $\mathrm{O}(21)-\mathrm{C}(22)-\mathrm{H}(22 \mathrm{a}) \quad 104.3(10)$ \\
\hline $\mathrm{N}(8)-\mathrm{C}(10)-\mathrm{C}(11)$ & 121.97(13) & $\mathrm{O}(21)-\mathrm{C}(22)-\mathrm{H}(22 \mathrm{~b}) \quad 109.1(10)$ \\
\hline $\mathrm{C}(10)-\mathrm{C}(11)-\mathrm{C}(12)$ & 110.2 & $\mathrm{O}(21)-\mathrm{C}(22)-\mathrm{H}(22 \mathrm{c}) \quad 110.5(10)$ \\
\hline $\mathrm{C}(10)-\mathrm{C}(11)-\mathrm{H}(11 \mathrm{a})$ & ) $110.5(9)$ & $\mathrm{H}(22 \mathrm{a})-\mathrm{C}(22)-\mathrm{H}(22 \mathrm{~b}) 112.0(14)$ \\
\hline $\mathrm{C}(10)-\mathrm{C}(11)-\mathrm{H}(11 \mathrm{~b})$ & $110.8(9)$ & $\mathrm{H}(22 \mathrm{a})-\mathrm{C}(22)-\mathrm{H}(22 \mathrm{c}) 109.2(14)$ \\
\hline $\mathrm{C}(12)-\mathrm{C}(11)-\mathrm{H}(11 \mathrm{a})$ & 107.4(10) & $\mathrm{H}(22 \mathrm{~b})-\mathrm{C}(22)-\mathrm{H}(22 \mathrm{c}) 111.6(14)$ \\
\hline $\mathrm{C}(12)-\mathrm{C}(11)-\mathrm{H}(11 \mathrm{~b})$ & $110.5(9)$ & $\mathrm{C}(12)-\mathrm{C}(23)-\mathrm{O}(24)$ \\
\hline & b) $107.2(13)$ & $\mathrm{C}(12)-\mathrm{C}(23)-\mathrm{O}(25)$ \\
\hline $\mathrm{C}(11)-\mathrm{C}(12)-\mathrm{C}(13)$ & $111.53(12)$ & $\mathrm{O}(24)-\mathrm{C}(23)-\mathrm{O}(25) \quad 124.14(14)$ \\
\hline$C(11)-C(12)-C(19)$ & $108.18(12)$ & $\mathrm{C}(23)-\mathrm{O}(25)-\mathrm{C}(26) \quad 115.94(14)$ \\
\hline $\mathrm{C}(11)-\mathrm{C}(12)-\mathrm{C}(23)$ & $109.73(12)$ & $\mathrm{O}(25)-\mathrm{C}(26)-\mathrm{H}(26 \mathrm{a}) \quad 109.5(13)$ \\
\hline $\mathrm{C}(13)-\mathrm{C}(12)-\mathrm{C}(19)$ & $112.57(12)$ & $\mathrm{O}(25)-\mathrm{C}(26)-\mathrm{H}(26 \mathrm{~b}) \quad 107.8(14)$ \\
\hline & 108.94(11) & $\mathrm{O}(25)-\mathrm{C}(26)-\mathrm{H}(26 \mathrm{c}) \quad 111.2(13)$ \\
\hline$C(19)-C(12)-C(23)$ & 105.71(11) & $\mathrm{H}(26 \mathrm{a})-\mathrm{C}(26)-\mathrm{H}(26 \mathrm{~b}) 106.4(19)$ \\
\hline
\end{tabular}


Table S6. Continued

$\begin{array}{llll}\mathrm{C}(12)-\mathrm{C}(13)-\mathrm{C}(14) & 108.93(11) & \mathrm{H}(26 \mathrm{a})-\mathrm{C}(26)-\mathrm{H}(26 \mathrm{c}) & 106.2(18) \\ \mathrm{C}(12)-\mathrm{C}(13)-\mathrm{C}(18) & 113.01(12) & \mathrm{H}(26 \mathrm{~b})-(26)-\mathrm{H}(26 \mathrm{c}) & 115.5(20) \\ \mathrm{C}(12)-\mathrm{C}(13)-\mathrm{H}(13) & 107.2(8) & \mathrm{C}(9)-\mathrm{H}(9 \mathrm{a})-\mathrm{H}(9 \mathrm{~b}) & 38.8(15) \\ \mathrm{C}(14)-\mathrm{C}(13)-\mathrm{C}(18) & 110.81(12) & \mathrm{C}(9)-\mathrm{H}(9 \mathrm{a})-\mathrm{H}(9 \mathrm{c}) & 34.8(14) \\ \mathrm{C}(14)-\mathrm{C}(13)-\mathrm{H}(13) & 109.8(8) & \mathrm{H}(9 \mathrm{~b})-\mathrm{H}(9 \mathrm{a})-\mathrm{H}(9 \mathrm{c}) & 61.9(18) \\ \mathrm{C}(18)-\mathrm{C}(13)-\mathrm{H}(13) & 107.0(8) & \mathrm{C}(9)-\mathrm{H}(9 \mathrm{~b})-\mathrm{H}(9 \mathrm{a}) & 38.4(15) \\ \mathrm{C}(1)-\mathrm{C}(14)-\mathrm{C}(13) & 108.67(11) & \mathrm{C}(9)-\mathrm{H}(9 \mathrm{c})-\mathrm{H}(9 \mathrm{a}) & 35.6(14) \\ \mathrm{C}(1)-\mathrm{C}(14)-\mathrm{C}(15) & 114.95(12) & \mathrm{C}(11)-\mathrm{H}(11 \mathrm{a})-\mathrm{H}(11 \mathrm{~b}) & 36.7(8) \\ \mathrm{C}(1)-\mathrm{C}(14)-\mathrm{H}(14) & 111.2(8) & \mathrm{C}(11)-\mathrm{H}(11 \mathrm{~b})-\mathrm{H}(11 \mathrm{a}) & 36.1(8)\end{array}$




\section{References}

1) Smith, A. B. III; Visnick, M.; Haseltine, J. N.; Sprengeler, P. A. Tetrahedron 1986, 42, 2957.

2) D'Ambra, T. E.; Estep, K. G.; Bell, M. R.; Eissenstat, M. A.; Josef, K. A.; Ward, S. J.;

Haycock, D. A.; Baizman, E. R.; Casiano, F. M.; et al. J. Med. Chem. 1992, 35, 124.

3) Pei, T.; Widenhoefer, R. A. J. Am. Chem. Soc. 2001. 125. 11290.

4) Ohe, T.; Miyaura, N.; Suzuki, A. J. Org Chem 1993, 58, 2201

5) Faul, M. M.; Winneroski, L. L. Tetrahedron Lett. 1997, 38, 4749.

6) Abbiati, G.; Beccalli, E. M.; Broggini, G.; Zoni, C. J. Org. Chem. 2003, 68, 7625.

7) a) Kantner, S.; Humski, K.; Goering, H. L. J. Am. Chem. Soc. 1982, 104, 1693. b)

Stiles, M.; Longroy, A. L. J. Org. Chem. 1967, 32, 1095.

8) Hoffmann, D.; Rathkamp, G.; Nesnow, S. Anal. Chem. 1969, 41, 1256.

9) Horning, E. C.; Horning, M. G.; Walker, G. N. J. Am. Chem. Soc. 1948, 70. 3935.

10) Murakami, Y; Yokoyama, Y; Okuyama, N. Tetrahedron Lett. 1983, 24, 2189.

11) Letcher, R. M.; Wai, J. S. M. J. Chem. Res. (S) 1986, 2, 37.

12) Commercially available from Exploratory Library.

13) (a) Merrifield, J. H.; Fernández, J. M.; Buhro, W. E.; Gladysz, J. A. Inorg. Chem. 1984, 23, 4022. (b) De Luca, N.; Wojcicki, A. J. Organomet. Chem. 1980, 193, 359. 